\title{
Advances in Antarctic Research for Antimicrobial Discovery: A Comprehensive Narrative Review of Bacteria from Antarctic Environments as Potential Sources of Novel Antibiotic Compounds Against Human Pathogens and Microorganisms of Industrial Importance
}

\author{
Kattia Núñez-Montero ${ }^{1,2,3}$ and Leticia Barrientos $1,2, *$ (D) \\ 1 Laboratorio de Biología Molecular Aplicada, Centro de Excelencia en Medicina Traslacional, Universidad \\ de La Frontera, Avenida Alemania 0458, 4810296 Temuco, Chile; k.nunez03@gmail.com \\ 2 Núcleo Científico y Tecnológico en Biorecursos (BIOREN), Universidad de La Frontera, Avenida Francisco \\ Salazar 01145, 481123 Temuco, Chile \\ 3 Centro de Investigación en Biotecnología, Escuela de Biología, Instituto Tecnológico de Costa Rica, \\ 30101 Cartago, Costa Rica \\ * Correspondence: leticia.barrientos@ufrontera.cl; Tel.: +56-452-592-802
}

Received: 13 July 2018; Accepted: 4 October 2018; Published: 19 October 2018

\begin{abstract}
The recent emergence of antibiotic-resistant bacteria has become a critical public health problem. It is also a concern for industries, since multidrug-resistant microorganisms affect the production of many agricultural and food products of economic importance. Therefore, discovering new antibiotics is crucial for controlling pathogens in both clinical and industrial spheres. Most antibiotics have resulted from bioprospecting in natural environments. Today, however, the chances of making novel discoveries of bioactive molecules from various well-known sources have dramatically diminished. Consequently, unexplored and unique environments have become more likely avenues for discovering novel antimicrobial metabolites from bacteria. Due to their extreme polar environment, Antarctic bacteria in particular have been reported as a potential source for new antimicrobial compounds. We conducted a narrative review of the literature about findings relating to the production of antimicrobial compounds by Antarctic bacteria, showing how bacterial adaptation to extreme Antarctic conditions confers the ability to produce these compounds. We highlighted the diversity of antibiotic-producing Antarctic microorganisms, including the phyla Proteobacteria, Actinobacteria, Cyanobacteria, Firmicutes, and Bacteroidetes, which has led to the identification of new antibiotic molecules and supports the belief that research on Antarctic bacterial strains has important potential for biotechnology applications, while providing a better understanding of polar ecosystems.
\end{abstract}

Keywords: Antarctic ecosystem; novel antibiotics; Antarctic bacteria; multidrug-resistant pathogens; extreme environment; bacterial cold-adaptation 


\section{Introduction}

The continual increase in global resistance to existing antibiotics, the prevalence of multidrug-resistant pathogens, and the rapid development of cross-resistance with new antibiotics have become a critical public health problem [1,2]. Bacterial antibiotic resistance can be acquired through mutation or horizontal transfer of a resistant gene, with phenotypic expression influenced by environmental and genetic factors [3]. All classes of antibiotics have experienced an emergence of resistance that compromises their use [4]. Hence, research efforts are aimed at discovering and producing novel and efficient therapeutic agents [5].

Regardless of efforts to produce new synthetic antibiotics [6], bioprospecting of natural environments has been responsible for the identification, production, and commercialization of most antibiotics [7] and it continues to provide key scaffolds for drug development [8]. Consequently, natural products-predominantly bacteria and fungi-are still the main sources for discovering novel antibiotics [7].

Recently, the chances of novel discoveries of bioactive molecules from various well-known bacteria have fallen dramatically. There are some hypotheses for this decrease in the discovery of new antibiotic molecules, including the large number of secondary metabolites that are cryptic or silent under common culture conditions, thereby inhibiting the identification of the total set of molecules from an isolate [9], and the frequent genetic exchanges between species that share similar chemical and physical selection factors in a particular environment $[10,11]$, making the chances of obtaining new molecules from the same environment more difficult. As a result, isolating highly bioactive Streptomyces strains-a common source of multiple antibiotics-from different and untapped environments is recommended for identifying new antibiotic molecules. The exploration of new, unexplored habitats and uncommon environments has therefore become important for discovering novel bacterial antimicrobial metabolites [7,12,13].

Extreme environments are unusual, yet promising sources of novel antibacterial compounds. Bacteria living in adverse environmental conditions display atypical survival strategies, such as antagonistic activity that reduces the presence of competitive microorganisms, in order to achieve competitive advantages [14]. Such behavior is particularly necessary when nutrients are limited or difficult to uptake. Antarctic microorganisms and other extremophiles could have developed physiologically unique features, including chemically complex biosynthesized metabolites, to ensure their survival in complex habitats, as it has been also exemplified for halotolerant marine microorganisms [15]. Moreover, genomic sequencing reveals that microbes with large genomes - usually inhabiting complex harsh environments [16] - have the capacity to produce about ten times as many secondary metabolites as was previously recognized [8].

The antagonistic properties of organisms inhabiting extreme environments have not been investigated as extensively as they have for mesophiles [17]; however, the traditional approach of isolating and cultivating new microorganisms from underexplored habitats is still productive [6]. In recent years, research has exploited untapped and extreme environments, including the polar regions, complex marine habitats, and hyperthermal environments, to discover novel bioactive compounds with antibacterial, antifungal, and antitumor properties, and apply them to current clinical challenges [15,18-21]. This review summarizes the findings from research on Antarctic bacteria producing antimicrobial compounds, in order to: (i) Highlight the importance of the Antarctic ecosystem as a promising source for discovering novel antibiotics, (ii) demonstrate the significance of a growing body of research focused on antimicrobial activity of Antarctic bacteria, and (iii) highlight the strains already isolated from different Antarctic regions and their potential application against human pathogens and phytopathogens and in other industrial and pharmaceutical applications. Literature search was no systemic, conducted through Web of Science databases (Core Collection and All Databases) using search parameters as follows: TOPIC $=\left(\right.$ antibiotic * or antimicrobial $\left.{ }^{*}\right)$ and (Antarctic or polar or extreme environment ${ }^{*}$ ). Articles where antimicrobial activity was reported from Antarctic bacterial strains were selected and included in this review. 


\section{The Antarctic Environment Supports Varied Microbial Life}

The Antarctic is separated from other continents by the Southern Ocean and the Antarctic Circumpolar Current [22]. It is a rocky land mass almost completely covered by an ice sheet, with regions of cold desert soils and freezing temperatures. Only about $2 \%$ of continental Antarctica is ice-free [23]. The massive ice sheet reflects $40-90 \%$ of the incidental solar radiation, causing a mass of cold dense air to accumulate on the polar plateau that sustains low polar temperatures. During the summer months, ice on the soils is subjected to thawing [24,25].

Consequently, Antarctica is known as the coldest, driest, windiest, and most inaccessible continent on the Earth [23]. In winter, temperatures at the South Pole reach a minimum of $-80^{\circ} \mathrm{C}$ to $-90{ }^{\circ} \mathrm{C}$ in the interior, and a maximum of $5^{\circ} \mathrm{C}$ to $15^{\circ} \mathrm{C}$ near the coast in summer. The higher elevation of east Antarctica makes it colder than its western counterpart [23]. Only cold-adapted organisms survive in Antarctica, including penguins, seals, nematodes, tardigrades, mites, tundra vegetation, and many types of algae and other microorganisms [23].

Although the Antarctic region has been considered to be a natural, unexploited environment, persistent pollutants have been reported, including heavy metals, antibiotics, pesticides that could have been transported through natural processes by air and water currents, as well as improper waste disposal practices and/or incineration from research stations [26,27]. Furthermore, recent studies show that scientists and tourists have introduced non-indigenous microorganisms to the Antarctic, with implications for natural genetic diversity, mainly due to the transference of antibiotic resistance [28,29]. Interestingly, active volcanoes have been confirmed as a local source of heavy metals (cadmium, zinc, vanadium, arsenic, and gold) [30]; however, the atmospheric transport of pollutants, such as mercury, continues to be considered a major pathway for Antarctic environment contamination [31,32].

The survival and growth of organisms in this region is restricted not only by low temperatures, but also by the availability of water and associated osmotic stress, substratum instability, and frequently introduced contaminants [24]. In spite of these conditions, prokaryotes are the dominant biomass component in most Antarctic ecosystems and play a major role in the food web, biogeochemical cycles, and mineralization of pollutants [33]. Microorganisms have been detected in all habitats, including lakes, ponds, rivers, streams, rocks, and soils [33].

Proteobacteria (a major phylum of Gram negative bacteria) have been frequently found in Antarctic soils [34]. Nevertheless, their habitats differ with respect to multiple physical-chemical parameters, including nutrients, water activity, range of temperature, and consequently, microbial flora [33]. Studies that focused on microbial diversity in Antarctic soils have reported Proteobacteria and Actinobacteria as the most abundant phyla, while the phyla Cyanobacteria and Firmicutes were common but less frequent [35-38]. Because Firmicutes comprise several spore-forming genera, it is highly reasonable to believe that this bacterial group is predominant in the harsh Antarctic environment, since it can survive under severe conditions for long periods of time [39].

To our knowledge, there are no studies to date representing the total microbial composition of the Antarctic. Nonetheless, a recent multi -omics approach (culture-independent technique) showed the prokaryotic community occurring within the active layer at Edmonson Point, an ice-free area on the eastern slope at the foot of Mount Melbourne (Northern Victoria Land, Antarctica) [40]. The composition of this area-from more to less abundant-showed Proteobacteria, Actinobacteria, Acidobacteria, Bacteroidetes, Planctomycetes, Chloroflexi, and Verrucomicrobia [40], while Proteobacteria, Actinobacteria, Bacteroidetes, and Firmicutes have been more frequently reported through cultivation-based methods $[17,34,41]$. Interestingly, 16 SrRNA sequencing and analysis revealed that anaerobic spore-forming Firmicutes have been shown to be the most abundant group in the Antarctic vascular plants rhizosphere [42].

Cultivable Antarctic bacteria have been frequently isolated using different media, depending on the group of interest, as was described for other environments, such as marine broth or agar for halotolerant [14], Luria-Bertani, tryptone soy [17,43], and R2A agar [34] for broad range culture. Diluted media, M1, oatmeal agar [44,45], among other culture media, have been recommended for 
the specific isolation of Actinobacteria. Even though Antarctic bacteria grow under low temperatures and survive freezing, optimal growth temperatures for isolates usually range from $4{ }^{\circ} \mathrm{C}$ to $20^{\circ} \mathrm{C}$, since Antarctic bacteria includes psychrotrophic and psychrophilic organisms [17]. Aerophyllic isolation conditions are usually preferred; therefore, aerobic organisms are typically isolated. However, microaerophilic bacterial strains, containing the anaerobic global regulator gene (anr), have been also reported [46], as well as obligate anaerobes [47,48].

Antarctic marine soils are free of snow and ice during the summer months and receive nutrients from the ocean and animal life-penguin guano, for example, enriches the soils with nitrogen and phosphorus, thus creating an environment capable of supporting abundant microbial growth [24]. $\mathrm{O}^{\prime}$ Brien et al. [17] also show that all ornithogenic soil samples yielded high bacterial counts (6-9 $\log$ CFU $\left.\cdot \mathrm{g}^{-1}\right)$ in comparison to other samples. Very high counts $\left(10 \mathrm{log} \mathrm{CFU} \cdot \mathrm{g}^{-1}\right)$ have been reported in ornithogenic soil through direct microscopic counting [49].

The presence of frozen water in Antarctic soils often leads to an increase in soil solute concentration, resulting in the creation of a hypersaline microenvironment [50]. Consequently, bacterial isolation in terms of colony-forming units from maritime Antarctica soils is higher in NaCl-containing media [17], showing the predominance of halotolerant bacteria in this habitat. In addition, high altitude soils in the Trans-Antarctic Mountains provide the least hospitable environment for microbial growth, since temperatures rise above freezing for only brief periods each year. Predictably, soils at over $1000 \mathrm{~m}$ above sea level contained low levels of microorganisms [17].

Although research on Antarctic microbial ecology began in the early 1900s [24], the region is still considered to be a potentially exploitable ecosystem for the discovery of new microorganisms with potential benefits for humans, due to its vast, unexplored diversity and peculiar environmental conditions. Bacteria with antimicrobial activity have been isolated from various Antarctic habitats, predominantly soils and seawater (Table 1). Higher antibiotic activity is typically produced by attached bacteria rather than their free-living counterparts in the pelagic zone [51,52]. This phenomenon might be due to the production of antimicrobial metabolites, which in a free-living stage could produce needless energy waste. Antagonistic interactions could play a key role in colonizing an ecological niche with high densities of bacteria [51,53], preventing other bacteria from colonizing particles. Although occurring less frequently, antimicrobial activity from free-living marine bacteria has also been reported and is reviewed in the following sections. 
Table 1. Antimicrobial substance-producing bacteria from Antarctic regions.

\begin{tabular}{|c|c|c|c|c|}
\hline Isolate Identification & Type of Sample & Place of Sampling & Antagonist Activity & Reference \\
\hline \multicolumn{5}{|l|}{ Actinobacteria } \\
\hline \multirow{5}{*}{ Arthrobacter sp. } & Sea water & $\begin{array}{l}72^{\circ} 19^{\prime} \mathrm{S} \text { to } 74^{\circ} 53^{\prime} \text { S }-163^{\circ} 48^{\prime} \text { E to } 70^{\circ} 16^{\prime} \text { E: Stations Mergellina Santa Maria } \\
\text { Novella, Tiburtina, Road Bay, Gerlache Inlet, Evans Cove, Inexpressible Island, } \\
\text { Cape Hallet, and Tethys Bay }\end{array}$ & $\begin{array}{l}\text { Escherichia coli, Micrococcus luteus, } \\
\text { Bacillus subtilis, Proteus mirabilis }\end{array}$ & [14] \\
\hline & Soil & $\begin{array}{l}72^{\circ} 19^{\prime} \text { S to } 77^{\circ} 83^{\prime} \text { S- } 16^{\circ} 55^{\prime} \text { E to } 17^{\circ} 16^{\prime} \text { E: Cape Hallett, Edmonson Point, Kay } \\
\text { Island, Cape Russell, Lake Hoare, Harrow Peaks, Crater Circe, Battleship } \\
\text { Promontory, Mount, McGee, Mount Rittmann, Mount Melbourne }\end{array}$ & Listeria spp., Brochothrix thermosphacta & [17] \\
\hline & Marine sediment & $74^{\circ} 41^{\prime} 36.96^{\prime \prime}$ S, $164^{\circ} 6^{\prime} 42.12^{\prime \prime}$ E: Terranova Bay, Ross Sea. & $\begin{array}{l}\text { Burkholderia cepacia complex } \\
\text { pathogens }\end{array}$ & [54] \\
\hline & Benthic microbial mat & Larsemann Hills, Vestfold Hills and McMurdo Dry Valleys & $\begin{array}{l}\text { Staphylococcus aureus, Enterococcus } \\
\text { faecium, E. coli }\end{array}$ & [55] \\
\hline & Ornithogenic soil & $\begin{array}{l}62^{\circ} 59^{\prime} \mathrm{S}, 60^{\circ} 34^{\prime} \mathrm{W} \text { : Whalers Bay on Deception Island, South Shetland Islands } \\
\text { and } 65^{\circ} 14^{\prime} 44.6^{\prime \prime} \mathrm{S}, 64^{\circ} 15^{\prime} 26^{\prime \prime} \mathrm{W} \text { : Galindez Island, Argentine Islands }\end{array}$ & $\begin{array}{l}\text { B. subtilis, Bacillus cereus, M. luteus, } \\
\text { Pseudomonas aeruginosa, Acinetobacter } \\
\text { johnsonii, Xanthomonas oryzae, Candida } \\
\text { sp., Cryptococcus sp. }\end{array}$ & [56] \\
\hline Brevibacterium sp. & Soil & $62^{\circ} 24^{\prime} \mathrm{S}, 59^{\circ} 47^{\prime} \mathrm{W}$ : Barrientos Island & S. aureus, Candida albicans & [57] \\
\hline Demetria sp. & Soil & $62^{\circ} 24^{\prime} \mathrm{S}, 59^{\circ} 47^{\prime} \mathrm{W}$ : Barrientos Island & S. aureus & [57] \\
\hline Gordonia sp. & Soil & $62^{\circ} 24^{\prime} \mathrm{S}, 59^{\circ} 47^{\prime} \mathrm{W}$ : Barrientos Island & S. aureus, C. albicans & [57] \\
\hline Gordonia terrae & Soil & $62^{\circ} 58^{\prime} 42.2^{\prime \prime} \mathrm{S}, 60^{\circ} 42^{\prime} 71.5^{\prime \prime} \mathrm{W}$ : Deception Island & $\begin{array}{l}\text { Salmonella enterica serotype Paratyphi, } \\
\text { S. enterica serotype Enteritidis }\end{array}$ & [58] \\
\hline Janibacter sp. & Soil & $62^{\circ} 24^{\prime} \mathrm{S}, 59^{\circ} 47^{\prime} \mathrm{W}$ : Barrientos Island & S. aureus & [57] \\
\hline Janibacter thuringensis & Sea water & $\begin{array}{l}72^{\circ} 19^{\prime} \mathrm{S} \text { to } 74^{\circ} 53^{\prime} \mathrm{S}-163^{\circ} 48^{\prime} \mathrm{E} \text { to } 70^{\circ} 16^{\prime} \mathrm{E} \text { : Stations Mergellina Santa Maria } \\
\text { Novella, Tiburtina, Road Bay, Gerlache Inlet, Evans Cove, Inexpressible Island, } \\
\text { Cape Hallet, and Tethys Bay }\end{array}$ & E. coli, M. luteus, P. mirabilis & [14] \\
\hline Kocuria sp. & Soil & $62^{\circ} 24^{\prime} \mathrm{S}, 59^{\circ} 47^{\prime} \mathrm{W}$ : Barrientos Island & S. aureus & [57] \\
\hline Lapillicoccus sp. & Soil & $62^{\circ} 24^{\prime} \mathrm{S}, 59^{\circ} 47^{\prime} \mathrm{W}$ : Barrientos Island & C. albicans & [57] \\
\hline Leifsonia soli & Soil & $62^{\circ} 58^{\prime} 42.2^{\prime \prime} \mathrm{S}, 60^{\circ} 42^{\prime} 71.5^{\prime \prime} \mathrm{W}$ : Deception Island & $\begin{array}{l}\text { S. enterica serotype Paratyphi, S. } \\
\text { enterica serotype Enteritidis }\end{array}$ & [58] \\
\hline Micromonospora sp. & Soil & $62^{\circ} 24^{\prime} \mathrm{S}, 59^{\circ} 47^{\prime} \mathrm{W}$ : Barrientos Island & S. aureus, $P$. aeruginosa & [57] \\
\hline Nesterenkonia sp. & Sea water & $\begin{array}{l}72^{\circ} 19^{\prime} \mathrm{S} \text { to } 74^{\circ} 53^{\prime} \mathrm{S}-163^{\circ} 48^{\prime} \mathrm{E} \text { to } 70^{\circ} 16^{\prime} \mathrm{E} \text { : Stations Mergellina Santa Maria } \\
\text { Novella, Tiburtina, Road Bay, Gerlache Inlet, Evans Cove, Inexpressible Island, } \\
\text { Cape Hallet, and Tethys Bay }\end{array}$ & E. coli & [14] \\
\hline \multirow{2}{*}{ Nocardioides sp. } & \multirow{2}{*}{ Soil } & Casey Station, Wilkes Land & S. aureus, B. subtilis, X. oryzae & [59] \\
\hline & & $62^{\circ} 24^{\prime} \mathrm{S}, 59^{\circ} 47^{\prime} \mathrm{W}$ : Barrientos Island & C. albicans & [57] \\
\hline
\end{tabular}


Table 1. Cont.

\begin{tabular}{|c|c|c|c|c|}
\hline Isolate Identification & Type of Sample & Place of Sampling & Antagonist Activity & Reference \\
\hline \multirow[b]{2}{*}{ Rhodococcus sp. } & Soil & $62^{\circ} 24^{\prime} \mathrm{S}, 59^{\circ} 47^{\prime} \mathrm{W}$ : Barrientos Island & S. aureus & [57] \\
\hline & Ornithogenic soil & $\begin{array}{l}62^{\circ} 59^{\prime} \mathrm{S}, 60^{\circ} 34^{\prime} \mathrm{W} \text { : Whalers Bay on Deception Island, South Shetland Islands } \\
\text { and } 65^{\circ} 14^{\prime} 44.6^{\prime \prime} \mathrm{S}, 64^{\circ} 15^{\prime} 26^{\prime \prime} \mathrm{W} \text { : Galindez Island, Argentine Islands }\end{array}$ & $\begin{array}{l}\text { B. subtilis, B. cereus, P. aeruginosa, } \\
\text { Candida sp., Cryptococcus sp. }\end{array}$ & [56] \\
\hline Rhodococcus fascians & Sea water & $\begin{array}{l}72^{\circ} 19^{\prime} \mathrm{S} \text { to } 74^{\circ} 53^{\prime} \mathrm{S}-163^{\circ} 48^{\prime} \mathrm{E} \text { to } 70^{\circ} 16^{\prime} \mathrm{E} \text { : Stations Mergellina Santa Maria } \\
\text { Novella, Tiburtina, Road Bay, Gerlache Inlet, Evans Cove, Inexpressible Island, } \\
\text { Cape Hallet, and Tethys Bay }\end{array}$ & E. coli, M. luteus, B. subtilis, P. mirabilis & [14] \\
\hline Streptomyces sp. & Soil & $62^{\circ} 12^{\prime} 26.4^{\prime \prime}$ S, $58^{\circ} 58^{\prime} 28.7^{\prime \prime}$ W: Fildes Peninsula, King George Island. & $\begin{array}{l}\text { Vibrio parahaemolyticus, S. enterica } \\
\text { serovar Typhimurium, Enterobacter } \\
\text { cloacae, Klebsiella pneumoniae, B. cereus, } \\
\text { Listeria monocytogenes (serotypes I, IV } \\
\text { and aa), Enterococcus. faecalis, } \\
\text { Staphylococcus haemolyticus, } \\
\text { Staphylococcus equorum, S. aureus }\end{array}$ & [60] \\
\hline Terrabacter lapilli & Soil & $62^{\circ} 58^{\prime} 42.2^{\prime \prime} \mathrm{S}, 60^{\circ} 42^{\prime} 71.5^{\prime \prime} \mathrm{W}$ : Deception Island & $\begin{array}{l}\text { S. enterica serotype Paratyphi, S. } \\
\text { enterica serotype Enteritidis }\end{array}$ & [58] \\
\hline \multicolumn{5}{|l|}{ Proteobacteria } \\
\hline Bradyrhizobium sp. & Soil & $62^{\circ} 24^{\prime} \mathrm{S}, 59^{\circ} 47^{\prime} \mathrm{W}$ : Barrientos Island & C. albicans & [57] \\
\hline Burkholderia sp. & Ornithogenic soil & $\begin{array}{l}62^{\circ} 59^{\prime} \mathrm{S}, 60^{\circ} 34^{\prime} \mathrm{W} \text { : Whalers Bay on Deception Island, South Shetland Islands } \\
\text { and } 65^{\circ} 14^{\prime} 44.6^{\prime \prime} \mathrm{S}, 64^{\circ} 15^{\prime} 26^{\prime \prime} \mathrm{W} \text { : Galindez Island, Argentine Islands }\end{array}$ & $\begin{array}{l}\text { B. subtilis, B. cereus, A. johnsonii, X. } \\
\text { oryzae. }\end{array}$ & [56] \\
\hline \multirow{4}{*}{ Janthinobacterium sp. } & Soil & $62^{\circ} 12^{\prime} \mathrm{S}, 58^{\circ} 57^{\prime}$ W: Fildes Peninsula, King George Island & $\begin{array}{l}\text { Acinetobacter baumannii, } \text { P. aeruginosa, } \\
\text { E. coli, K. pneumoniae, Serratia } \\
\text { marcescens }\end{array}$ & [61] \\
\hline & Freshwater & $\begin{array}{l}70^{\circ} 45^{\prime} 52.3^{\prime \prime} \mathrm{S}, 11^{\circ} 37^{\prime} 10.7^{\prime \prime} \text { E: Lake Podprudnoye, Schirmacher Oasis, Dronning } \\
\text { Maud Land }\end{array}$ & $\begin{array}{l}\text { Mycobacterium smegmatis, } \\
\text { Mycobacterium tuberculosis }\end{array}$ & [62] \\
\hline & Benthic microbial mat & Larsemann Hills, Vestfold Hills and McMurdo Dry Valleys & S. aureus, E. faecium, E. coli & [55] \\
\hline & Ornithogenic soil & $\begin{array}{l}62^{\circ} 59^{\prime} \mathrm{S}, 60^{\circ} 34^{\prime} \mathrm{W} \text { : Whalers Bay on Deception Island, South Shetland Islands } \\
\text { and } 65^{\circ} 14^{\prime} 44.6^{\prime \prime} \text { S, } 64^{\circ} 15^{\prime} 26^{\prime \prime} \mathrm{W} \text { : Galindez Island, Argentine Islands }\end{array}$ & $\begin{array}{l}\text { B. subtilis, B. cereus, P. aeruginosa, E. } \\
\text { coli, Candida sp., Cryptococcus sp. }\end{array}$ & [56] \\
\hline Halomonas sp. & Sea water & $\begin{array}{l}57^{\circ} 59^{\prime} 422^{\prime \prime} \text { to } 64^{\circ} 33^{\prime} 779^{\prime \prime} \text { S }-45^{\circ} 27^{\prime} 440^{\prime \prime} \text { to } 63^{\circ} 16^{\prime} 554^{\prime \prime} \text { : Antarctic Peninsula and } \\
\text { South Shetland Islands area }\end{array}$ & $\begin{array}{l}\text { E. coli, S. enterica, Enterobacter } \\
\text { aerogenes, Citrobacter freundii, S. } \\
\text { marcescens, Shigella sp., S. aureus, } \\
\text { Staphylococcus epidermidis, B. subtilis, } \\
\text { Xanthomonas sp., Erwinia sp. }\end{array}$ & [63] \\
\hline Lysobacter oligotrophicus & $\begin{array}{l}\text { Bottom of freshwater } \\
\text { lake }\end{array}$ & Skarvsnes region & $\begin{array}{l}\text { E. coli, Lysobacter enzymogenes, } \\
\text { Rhodoligotrophos appendicifer, } \\
\text { Saccharomyces cerevisiae. }\end{array}$ & [64] \\
\hline Methylobacterium sp. & Soil & $62^{\circ} 24^{\prime} \mathrm{S}, 59^{\circ} 47^{\prime} \mathrm{W}$ : Barrientos Island & S. aureus, C. albicans & [57] \\
\hline Paracoccus sp. & Soil & $62^{\circ} 24^{\prime} \mathrm{S}, 59^{\circ} 47^{\prime} \mathrm{W}$ : Barrientos Island & C. albicans & [57] \\
\hline
\end{tabular}


Table 1. Cont.

\begin{tabular}{|c|c|c|c|c|}
\hline Isolate Identification & Type of Sample & Place of Sampling & Antagonist Activity & Reference \\
\hline \multirow{2}{*}{ Pseudoalteromonas sp. } & \multirow{2}{*}{ Sea water } & \multirow{2}{*}{$\begin{array}{l}72^{\circ} 19^{\prime} \mathrm{S} \text { to } 74^{\circ} 53^{\prime} \mathrm{S}-163^{\circ} 48^{\prime} \mathrm{E} \text { to } 70^{\circ} 16^{\prime} \mathrm{E} \text { : Stations Mergellina Santa Maria } \\
\text { Novella, Tiburtina, Road Bay, Gerlache Inlet, Evans Cove, Inexpressible Island, } \\
\text { Cape Hallet, and Tethys Bay }\end{array}$} & E. coli, M. luteus, P. mirabilis. & [14] \\
\hline & & & B. cepacia complex pathogens & [65] \\
\hline \multirow{2}{*}{$\begin{array}{l}\text { Pseudoalteromonas } \\
\text { haloplanktis }\end{array}$} & \multirow{2}{*}{ Sea water } & \multirow{2}{*}{$66^{\circ} 40^{\prime}$ S; $140^{\circ} 01^{\prime}$ E: French Antarctic station Dumont d' Urville, Terre Adélie } & Biofilms formation of S. epidermidis & [66] \\
\hline & & & B. cepacia complex pathogens & {$[67,68]$} \\
\hline \multirow{6}{*}{ Pseudomonas sp. } & Soil & $\begin{array}{l}72^{\circ} 19^{\prime} \mathrm{S} \text { to } 77^{\circ} 83^{\prime} \mathrm{S}, 16^{\circ} 55^{\prime} \mathrm{E} \text { to } 17^{\circ} 16^{\prime} \mathrm{E} \text { : Cape Hallett, Edmonson Point, Kay } \\
\text { Island, Cape Russell, Lake Hoare, Harrow Peaks, Crater Circe, Battleship } \\
\text { Promontory, Mount, McGee, Mount Rittmann, Mount Melbourne }\end{array}$ & Listeria spp., B. thermosphacta & [17] \\
\hline & Sub-sea sediment & $74^{\circ} 41^{\prime} 36.96^{\prime} \mathrm{S}, 164^{\circ} 6^{\prime} 42.12^{\prime}$ E: Terranova Bay, Ross sea & B. cepacia complex pathogens & [54] \\
\hline & Marine sediment & $\begin{array}{l}62^{\circ} 58^{\prime} 788^{\prime \prime} \text { to } 62^{\circ} 05^{\prime} 948^{\prime \prime} \mathrm{S}-60^{\circ} 33^{\prime} 464^{\prime \prime} \text { to } 58^{\circ} 23^{\prime} 622^{\prime \prime} \mathrm{W} \text { : } \\
\text { Deception Island, Martel Bay, King George Island and Punta Hannah sediment }\end{array}$ & M. luteus, S. aureus & [38] \\
\hline & Benthic microbial mat & Larsemann Hills, Vestfold Hills and McMurdo Dry Valleys & S. aureus, E. faecium, E. coli & [55] \\
\hline & Ornithogenic soil & $\begin{array}{l}62^{\circ} 59^{\prime} \text { S, } 60^{\circ} 34^{\prime} \text { W: Whalers Bay on Deception Island, South Shetland Islands } \\
\text { and } 65^{\circ} 14^{\prime} 44.6^{\prime \prime} \text { S, } 64^{\circ} 15^{\prime} 26^{\prime \prime} \text { W: Galindez Island, Argentine Islands }\end{array}$ & $\begin{array}{l}\text { B. subtilis, B. cereus, Sarcina lutea, } M \text {. } \\
\text { luteus } P . \text { aeruginosa, E. coli, A. johnsonii, } \\
\text { X. oryzae, Candida sp., Cryptococcus sp. }\end{array}$ & [56] \\
\hline & Soil & $62^{\circ} 09^{\prime} 30.0^{\prime \prime}$ S, $58^{\circ} 56^{\prime} 15.2^{\prime \prime} \mathrm{W}$ : King George Island & $\begin{array}{l}\text { E. coli, S. enterica serovar Typhimurium, } \\
\text { K. pneumoniae, E. cloacae, B. cereus, } V \text {. } \\
\text { parahaemolyticus }\end{array}$ & [69] \\
\hline Pseudomonas fragi & $\begin{array}{l}\text { Water column, rock } \\
\text { surfaces }\end{array}$ & $62^{\circ} 12^{\prime} \mathrm{S}, 58^{\circ} 57^{\prime} \mathrm{W}$ : King George Island & Flavobacterium psychrophilum biofilm & [70] \\
\hline \multirow{2}{*}{ Psychrobacter } & Sub-sea sediment & $74^{\circ} 41^{\prime} 36.96^{\prime \prime} \mathrm{S}, 164^{\circ} 6^{\prime} 42.12^{\prime \prime}$ E: Terranova Bay, Ross sea & B. cepacia complex pathogens & [54] \\
\hline & Benthic microbial mat & Larsemann Hills, Vestfold Hills and McMurdo Dry Valleys & S. aureus, E. coli & [55] \\
\hline Shewanella sp. & $\begin{array}{l}\text { Lake ponds benthic } \\
\text { microbial mats }\end{array}$ & Larsemann Hills, Vestfold Hills andMcMurdo Dry Valleys & S. aureus, E. coli & [55] \\
\hline Sphingomonas sp. & Soil & $62^{\circ} 24^{\prime} \mathrm{S}, 59^{\circ} 47^{\prime} \mathrm{W}$ : Barrientos Island & C. albicans & [57] \\
\hline \multicolumn{5}{|l|}{ Cyanobacteria } \\
\hline Leptolyngbya antarctica & Benthic microbial mat & $\begin{array}{l}\text { Larsemann Hills, Bølingen Islands, Vestfold Hills, Rauer Islands, and the } \\
\text { McMurdo Dry Valleys }\end{array}$ & S. aureus & [71] \\
\hline Nostoc sp. & Benthic microbial mat & $\begin{array}{l}\text { Larsemann Hills, Bølingen Islands, Vestfold Hills, Rauer Islands, and the } \\
\text { McMurdo Dry Valleys }\end{array}$ & $\begin{array}{l}\text { M. tuberculosis, S. aureus, S. typhi, P. } \\
\text { aeruginosa, E. aerogenes, } \\
\text { multidrug-resistant strains of E. coli, } \\
\text { C. neoformans }\end{array}$ & {$[71,72]$} \\
\hline Phormidium priestleyi & Benthic microbial mat & $\begin{array}{l}\text { Larsemann Hills, Bølingen Islands, Vestfold Hills, Rauer Islands, and the } \\
\text { McMurdo Dry Valleys }\end{array}$ & $\begin{array}{l}\text { S. aureus, A. fumigatus, Cryptococcus } \\
\text { neoformans }\end{array}$ & [71] \\
\hline Phormidium murrayi & Benthic microbial mat & $\begin{array}{l}\text { Larsemann Hills, Bølingen Islands, Vestfold Hills, Rauer Islands, and the } \\
\text { McMurdo Dry Valleys }\end{array}$ & S. aureus & [71] \\
\hline
\end{tabular}


Table 1. Cont

\begin{tabular}{|c|c|c|c|c|}
\hline Isolate Identification & Type of Sample & Place of Sampling & Antagonist Activity & Reference \\
\hline Pseudophormidium sp. & Benthic microbial mat & $\begin{array}{l}\text { Larsemann Hills, Bølingen Islands, Vestfold Hills, Rauer Islands, and the } \\
\text { McMurdo Dry Valleys }\end{array}$ & S. aureus, C. neoformans & [71] \\
\hline \multicolumn{5}{|l|}{ Firmicutes } \\
\hline \multirow{3}{*}{ Bacillus sp. } & Sea water & $50^{\circ} 76^{\prime} \mathrm{W}, 61^{\circ} 16^{\prime} \mathrm{S}$ & $\begin{array}{l}\text { Paecilomyces variotii, Colletotrichum } \\
\text { gloeosporioides, Fusarium oxysporum, } \\
\text { Trichoderma viride, Rhizoctonia solani }\end{array}$ & [73] \\
\hline & Ornithogenic soil & $\begin{array}{l}62^{\circ} 59^{\prime} \mathrm{S}, 60^{\circ} 34^{\prime} \mathrm{W} \text { : Whalers Bay on Deception Island, South Shetland Islands } \\
\text { and } 65^{\circ} 14^{\prime} 44.6^{\prime} \mathrm{S}, 64^{\circ} 15^{\prime} 26^{\prime \prime} \mathrm{W} \text { : Galindez Island, Argentine Islands }\end{array}$ & $\begin{array}{l}\text { B. subtilis, B. cereus, P. aeruginosa, E. } \\
\text { coli, A. johnsonii, Candida sp. }\end{array}$ & [56] \\
\hline & Soil & $62^{\circ} 04^{\prime} \mathrm{S}, 58^{\circ} 21^{\prime} \mathrm{W}$ : King George Island & $\begin{array}{l}\text { Methicillin resistant } S \text {. aureus, } C \text {. } \\
\text { albicans }\end{array}$ & [74] \\
\hline Enterococcus sp. & Soil & $\begin{array}{l}69^{\circ} 21.68^{\prime} \mathrm{S}, 76^{\circ} 07.76^{\prime} \mathrm{E} ; 69^{\circ} 21.68^{\prime} \mathrm{S}, 76^{\circ} 07.70^{\prime} \mathrm{E} \text { and } 69^{\circ} 22.433^{\prime} \mathrm{S}, 76^{\circ} 08.940^{\prime} \mathrm{E}: \\
\text { Penguin rookeries Larsemann Hills, East Antarctica }\end{array}$ & $\begin{array}{l}\text { Multidrug-resistant strains of } C \text {. } \\
\text { albicans }\end{array}$ & [75] \\
\hline Planococcus sp. & Soil & $\begin{array}{l}72^{\circ} 19^{\prime} \mathrm{S} \text { to } 77^{\circ} 83^{\prime} \mathrm{S}-16^{\circ} 55^{\prime} \mathrm{E} \text { to } 17^{\circ} 16^{\prime} \mathrm{E} \text { : Cape Hallett, Edmonson Point, Kay } \\
\text { Island, Cape Russell, Lake Hoare, Harrow Peaks, Crater Circe, Battleship } \\
\text { Promontory, Mount, McGee, Mount Rittmann, Mount Melbourne }\end{array}$ & Listeria spp., B. thermosphacta & [17] \\
\hline \multirow[t]{2}{*}{ Sporosarcina sp. } & \multirow[t]{2}{*}{ Ornithogenic soil } & $\begin{array}{l}62^{\circ} 59^{\prime} \mathrm{S}, 60^{\circ} 34^{\prime} \mathrm{W} \text { : Whalers Bay on Deception Island, South Shetland Islands } \\
\text { and } 65^{\circ} 14^{\prime} 44.6^{\prime \prime} \mathrm{S}, 64^{\circ} 15^{\prime} 26^{\prime \prime} \mathrm{W} \text { : Galindez Island, Argentine Islands }\end{array}$ & $\begin{array}{l}\text { B. subtilis, } B . \text { cereus, S. lutea, } P \text {. } \\
\text { aeruginosa, E. coli, A. johnsonii, X. } \\
\text { oryzae, Candida sp., Cryptococcus sp. }\end{array}$ & [56] \\
\hline & & $62^{\circ} 04^{\prime} \mathrm{S}, 58^{\circ} 21^{\prime} \mathrm{W}$ : King George Island & $\begin{array}{l}\text { Methicillin resistant } S \text {. aureus, } C \text {. } \\
\text { albicans }\end{array}$ & [74] \\
\hline \multicolumn{5}{|l|}{ Bacteroidetes } \\
\hline Flavobacterium sp. & Freshwater & $\begin{array}{l}70^{\circ} 45^{\prime} 52.3^{\prime \prime} \text { S, } 11^{\circ} 37^{\prime} 10.7^{\prime \prime} \text { E: Lake Podprudnoye, Schirmacher Oasis, Dronning } \\
\text { Maud Land }\end{array}$ & M. smegmatis, M. tuberculosis & [62] \\
\hline Pedobacter sp. & Soil & $62^{\circ} 09^{\prime} 30.0^{\prime \prime}$ S, 58 56 $56^{\prime} 15.2^{\prime \prime}$ W: King George Island & $\begin{array}{l}\text { E. coli, S. enterica serovar Typhimurium, } \\
\text { S. enterica serovar Typhi, K. pneumoniae, } \\
\text { E. cloacae, B. cereus }\end{array}$ & [69] \\
\hline
\end{tabular}




\section{Bacterial Adaptation to Antarctic Extreme Conditions Might Confer the Ability to Produce Diverse and Novel Antimicrobial Compounds}

Due to their extreme environment, Antarctic bacteria have been reported as a potential source for antimicrobial compounds [61]. Microorganisms need to develop different strategies to survive polar conditions [76,77], such as lack of substrate, high UV-radiation, sustained low temperatures, and short-term intense heat during the Antarctic summer [23]. These complex conditions represent diverse challenges for bacterial life, including reduced enzyme activity, decreased membrane fluidity, altered transport of nutrients and waste products, decreased rates of transcription, translation, and cell division, protein cold-denaturation, inappropriate protein folding, and intracellular ice formation [78].

Therefore, genetic adaptation of Antarctic microorganisms to polar stress factors would be expected to generate novel metabolic pathways through evolutionary selection [76]. Thus, one can suggest the synthesis of new metabolites from Antarctic bacteria with unique structures and specific biological activity, produced as a competitive advantage for niche colonization by establishing antagonistic relationships [23].

Such antagonistic interactions among Antarctic bacteria have been demonstrated [79], and numerous Antarctic bacterial isolates exhibited antagonistic properties against marine bacteria from the same environment [80]. Differences were observed in the inhibition patterns of single isolates, suggesting that their activity was more likely strain-specific rather than dependent on phylogenetic affiliation [79]. Similarly, five Actinobacteria strains were isolated from surfaces of Antarctic macroalgae and showed activity against other macroalgae-resident bacteria, both Gram-negative and Gram-positive strains [80]. This data suggest the potential exploitation of Antarctic bacteria as a novel source of antimicrobial compounds and confirms antagonistic interactions within bacterial strains in Antarctic marine ecosystems.

Reports on Pseudoalteromonas spp. also support the case for discovering Antarctic bacteria suitable for the production of antimicrobial metabolites. This genus has become a model for studying bacterial adaptation to cold, since it is frequently isolated from marine polar regions [81-83]. Genomic analysis of the Antarctic strain Pseudoalteromonas haloplanktis TAC125 has demonstrated important features relating to its adaptability [84]. Among them a specific codon usage bias, which is involved in the resistance to protein aging features including asparagine cyclization and deamination [85]; the resistance to reactive oxygen species (ROS), which represent a significant stressor under cold conditions [86,87]; and the elevated number of rRNA and tRNA genes, which could explain its translational efficiency even in cold conditions [67]. Additionally, cryotolerance and osmotic stress resistance have been conferred by the enhanced uptake of compounds including: Spermine, Glutathione, Ornithine, and others, related to Glutathione metabolism; increased metabolic potential in terms of Arginine and Proline metabolism, $\beta$-Alanine metabolism, fatty acid uptake, and biosynthesis; and protein S-thiolation [88]. Similarly, carotenoid pigmentation confers protection to Antarctic bacteria against freeze-thaw cycles and solar radiation [89].

Another approach using the existing genomes of Pseudoalteromonas isolates has shown important evolutionary implications regarding the diverse genetic information of cold-adapted bacteria, including the relatively high number of genes obtained through horizontal gene transfer-12,981 genes (14\% of the genome) were associated with mobile genetic elements-and an extensive evolutionary genome reduction of this genus [84]. These modifications probably acted as the main factors leading to the genomic diversity between Pseudoalteromonas strains, and might correspond to other isolated Antarctic bacteria.

On the other hand, it is reasonable to believe that strains isolated from the Antarctic might possess genes involved in the adaptation to cold temperatures, such as Anti-Freeze Proteins (AFPs), a class of proteins able to inhibit ice nucleation or decrease the water freezing point temperature [90,91]. Remarkably, recent studies revealed no clear distinction between Antarctic and non-Antarctic Pseudoalteromonas strains, with all of them showing four to five AFPs [84]. This could be a genus-specific feature. 
Regarding antimicrobial compounds, Bosi et al. [84] demonstrated that Antarctic Pseudoalteromonas genomes harbor at least one cluster involved in bioactive molecule synthesis, with the number of clusters per genome ranging from 1 to 19 . As expected, high variability of biosynthetic operons was confirmed in terms of number and composition across the different genomes [84]. Furthermore, experimental evidence has shown the production of broad-spectrum antimicrobial compounds by Antarctic Actinobacteria preferentially at lower temperatures [60]. This behavior and genetic evidence supports the assumptions that Antarctic bacteria use antimicrobial compounds as an advantage to compete for nutrients and space in the cold environment.

\section{Antarctic Exhibits Significant Diversity of Antibiotic-Producing Bacteria with Pharmaceutical Applications}

\subsection{Proteobacteria Phylum Members}

A screening for bioactive secondary metabolites showed that 25\% (14 out of 57 ) of Proteobacterium species isolated from Antarctic soils generate an extracellular crude extract that is highly bioactive against Candida albicans and Staphylococcus aureus. The most bioactive genus was Bradyrhizobium, which contributed $64 \%$ of the total bioactive isolates in the study (9 out of 14) [11]. However, genera Methylobacterium, Paracoccus, and Sphingomonas were bioactive as well [11]. Other screenings also report the antimicrobial activity of Halomonas sp. strains against a broad range of human pathogens, including the Gram negative Salmonella enterica, Escherichia coli, Enterobacter aerogenes, Citrobacter freundii, Serratia marcescens, Shigella flexnerii, Shigella sonnei, Staphylococcus aureus, and Staphylococcus epidermidis, and the Gram positive Enterococcus faecalis and Listeria monocytogenes [63].

Asencio et al. [61] reported on a Janthinobacterium sp. ethanolic extract presenting antibacterial activity against multidrug-resistant bacteria isolates from Chilean hospitals. Among them were Acinetobacter baumannii and Pseudomonas aeruginosa, both carbapenemase producers; E. coli and Klebsiella pneumoniae, producing extended-spectrum $\beta$-lactamases; and two multiresistant strains of Serratia marcescens, producing a chromosomal AmpC beta-lactamase (the MIC values ranged between 0.5 and $16 \mu \mathrm{g} \cdot \mathrm{mL}^{-1}$ ) [61]. Janthinobacterium sp. extract activity against Mycobacterium smegmatis, Mycobacterium tuberculosis [62], Bacillus subtilis, Bacillus cereus, Candida sp., and Cryptococcus sp. [56] have been reported as well.

Similarly, members of Pseudomonas genus were found to produce proteinaceous compounds active against Gram positive bacteria (Listeria spp., B. subtilis, Bacillus cereus, Sarcina lutea, Micrococcus luteus, and Brochothrix thermosphacta), Gram negative bacteria (P. aeruginosa, E. coli, Acinetobacter johnsonii, S. enterica, K. pneumoniae, Enterobacter cloacae, and Vibrio parahaemolyticus) and yeast (Candida sp., Cryptococcus sp.) $[17,56,69]$. Another Pseudomonas sp. strain isolated from marine sediments showed activity against Staphylococcus aureus [38]. Additionally, volatile and soluble compounds against numerous members of Burkholderia cepacia complex (Bcc) pathogens have been reported for other Pseudomonas sp. isolates [54]. This activity was also described for Psychrobacter sp. strains obtained from marine sediments [54]. Interestingly, a Burkholderia sp. strain isolated from ornithogenic soil showed antimicrobial potential against some Gram positive (B. subtilis and B. cereus) and Gram negative (A. johnsonii) bacteria [56].

Strains belonging to Pseudoalteromonas genus expressed antagonistic activity against E. coli and Proteus mirabilis [14] and several members of Bcc [65], inhibiting their growth primarily due to the synthesis of numerous volatile compounds (VOCs). Remarkably, this study reported a constitutive synthesis of the volatile antimicrobial compounds (not induced by the presence of target strains), suggesting that production of such molecules is a common feature for the Antarctic strains [65]. Additionally, P. haloplanktis TAC125 produces a saccharidic compound that lacks antibacterial activity against free-living bacteria, but exhibits specific activity against biofilms formation of $S$. epidermidis strains isolated from infected catheter and septic arthritis [66]. Other studies have identified antibiotic compounds from different Pseudoalteromonas sp. strains isolated from marine environments, including 
extracellular active agents [92], molecules against methicillin-resistant pathogens [93], and genetic clusters related to the synthesis and regulation of antimicrobial compounds [94].

Since different Pseudoalteromonas sp. strains have shown important bioactive characteristics, this taxon is used as a model for molecular studies on the potential of Antarctic bacteria. In particular, Antarctic isolates have a great number of biosynthetic operons producing antimicrobial compounds, and pigmented Pseudoalteromonas sp. strains are characterized by the presence of a higher number of operons [84]. This result agrees with bioactivity assays where pigmented Pseudoalteromonas sp. exhibited the largest inhibition [95]. Other authors have highlighted the antimicrobial potential of pigmented bacteria from Antarctic marine environments [80]. Consequently, it has been proposed that those strains are able to produce a wider array of bioactive molecules, since they have a larger number of antimicrobial-producing operons [84].

Additionally, novel Proteobacteria species with antimicrobial activity have been found in the Antarctic. Lysobacter oligotrophicus was first described as a new member of the genus Lysobacter, which are strongly proteolytic and characteristically lyse a variety of microorganisms, as well as nematodes. $L$. oligotrophicus produced esterase, amylase, and protease, and showed a capacity to lyse Gram negative bacteria (E. coli, Lysobacter enzymogenes, and Rhodoligotrophos appendicifer) and Saccharomyces cerevisiae. Indeed, autolysis of this strain was observed in the stationary phase [64].

\subsection{Actinobacteria Phylum Members}

It is well known that bacteria from the phylum Actinobacteria represent a prominent source of biologically active natural compounds, since they produce versatile secondary metabolites [8]. Moreover, Actinobacteria are one of the major phyla of the domain bacteria, comprising 17 orders [96,97]. Remarkably, Actinobacteria comprise the genus Mycobacterium (a deadly bacterial pathogen), but also Streptomyces genus, which are most important for antibiotic production and are responsible for two-thirds of all known antibiotics [98].

Katz and Baltz [8] reported that bacteria belonging to this phylum have the capacity to produce around 30 to 50 secondary metabolites, making them one of the major sources of natural compounds. However, in recent years, the discovery of new antibiotic molecules from Actinobacteria has been infrequent, in part due to the recurrent genetic exchange between species from similar ecosystems [10]. As a result, the latest studies are focused on a search for Actinobacteria from unusual and isolated environments in order to discover novel antimicrobial metabolites [99,100].

Antarctic investigations have shown promising results with antimicrobial active compounds from Actinobacteria. A Streptomyces sp. strain capable of inhibiting the growth of seven Gram negative food borne pathogens (from genera Vibrio, Enterobacter, Klebsiella and Salmonella) and eight Gram positive food borne pathogens (from genera Listeria, Bacillus, Enterococcus and Staphylococcus) was isolated from Antarctic soil [60]. Likewise, strains of Arthrobacter sp. were found to produce active compounds against a broad range of human pathogens, including Gram positive, Gram negative, yeast (B. subtilis, B. cereus, M. luteus, P. aeruginosa, A. johnsonii, Candida sp., Cryptococcus sp., Listeria spp. and B. thermosphacta) $[17,56]$ and several Bcc strains [54]. Moreover, 22 marine Antarctic strains belonging predominantly to Arthrobacter sp., Janibacter thuringensis, Nesterenkonia sp., and Rhodococcus fascians expressed antagonistic activity against E. coli, M. luteus, B. subtilis, and P. mirabilis [14]. Similar results have been reported for other Rhodococcus sp. strains isolated from Antarctic ornithogenic soils [56].

Lee et al. (2012) [57] reported extracellular bioactive metabolites from 38\% (15 out of 39) of Actinobacterial isolates, inhibiting the growth of $C$. albicans, methicillin-resistant $S$. aureus, and $P$. aeruginosa. Brevibacterium genus showed the highest activity, with five bioactive isolates [57]. Although the 15 isolates with antibiotic activity were obtained from soil samples at the same location, the isolates turned out to be taxonomically diverse, since the strains belong to 11 different genera (Nocardioides, Gordonia, Micromonospora, Demetria, Rhodococcus, Janibacter, Dermacoccus, Kocuria, Lapillicoccus, and Brevibacterium). These findings confirm the abundant bacterial diversity in Antarctic environments. Comparably, Gordonia terrae, Leifsonia soli, and Terrabacter lapilli isolated from Antarctic volcanic soils 
showed inhibitory activity against $S$. enterica serotype Paratyphi and S. enterica serotype Enteritidis [58]. Other marine Actinobacteria have been reported to have antimicrobial activity against numerous human pathogens. In fact, novel species have been found to exhibit at least one of the biosynthetic genes coding for polyketide synthases and non-ribosomal peptide synthetases [18] related to the production of these secondary metabolites [101].

Furthermore, Nocardioides sp. belonging to Actinobacteria phylum isolated from Antarctic soil showed extracellular antimicrobial activity against Gram positive and Gram negative bacteria, especially S. aureus and B. subtilis. Preliminary analysis showed that the compounds with antimicrobial activity produced by this strain are mainly glycolipids and/or lipopeptides. Remarkably, the type of compound and specific activity against $S$. aureus depended on the used carbon source [59]. This finding is important because the antimicrobial substances might be produced mainly by the secondary metabolite pathway, depending on nutritional conditions and probably based on different chemical signals from the environment, as reported for other Antarctic isolates [55,68,102]. Hence, nutritional factors, such as carbon and nitrogen sources, metal ions, and inorganic phosphate in the media, may affect the production of secondary metabolites during cultivation of the microorganisms. This could be a critical aspect to consider for screening and discovering antimicrobial compounds produced by bacteria.

\subsection{Other Phylum Members}

A dominance of Cyanobacterial diversity in Antarctic and other polar environments has been reported by Vincent [103] and Zakhia et al. [104], indicating that the search for antimicrobial compounds from Antarctic Cyanobacteria seems to have a promising future. Interestingly, novel Antarctic Cyanobacteria was described as having antibacterial/antifungal potential. Some unknown and uncultured Cyanobacterial strains with antibiotic activity were found through $16 \mathrm{~S}$ ribosomal RNA gene (16S rRNA) and ITS sequence analysis [104]. From 48 total isolates, 35\% of the strains (identified as Pseudophormidium sp., Phormidium priestleyi, Leptolyngbya antarctica, Nostoc sp. and Phormidium murrayi) showed activity against $S$. aureus, or the fungi Aspergillus fumigatus and Cryptococcus neoformans in a strain-specific manner, using ethyl acetate and methanol crude extracts [71,105]. Activity against $M$. tuberculosis, S. aureus, S. typhi, P. aeruginosa, Enterobacter aerogenes and multidrug-resistant strains of $E$. coli, was also reported for extracts of Antarctic Nostoc sp. strains [72].

On the other hand, bioactivity of Firmicutes phylum has been occasionally reported from Antarctic isolates; however, $\mathrm{O}^{\prime} \mathrm{Brien}$ et al. [17] reported for the first time the isolation of Planococcus sp. strains from Antarctic soil with the production of bioactive compounds against Gram positive human pathogens (Listeria spp. and B. thermosphacta). In addition, a proteinaceous compound from Enterococcus sp. isolated from penguin rookeries and belonging to Firmicutes phylum exhibited a strong effect against multidrug-resistant strains of C. albicans [75]. Bacillus sp. and Sporosarcina sp. strains isolated from Antarctic soils demonstrated activity against yeast (Candida sp. and Cryptococcus sp.), Gram positive (B. subtilis, B. cereus, S. lutea, and methicillin resistant S. aureus) and Gram negative bacteria (P. aeruginosa, E. coli, and A. johnsonii) [56,74].

Similarly, Mojib et al. [62] first described an Antarctic Flavobacterium sp. belonging to Bacteroidetes phylum, with activity against M. smegmatis and M. tuberculosis. In addition, an Antarctic Pedobacter sp. strain, a member of this phylum, was reported for the first time as producing inhibitors against human pathogens (E. coli, S. enterica, K. pneumoniae, E. cloacae, and B. cereus) [69]. Additionally, in a high throughput screening for antimicrobial activity, 675 strains were isolated from 17 samples of benthic mats collected from 10 lakes located in three distinct Antarctic regions and 18\% were active against at least one of the panel pathogens (S. aureus, E. faecium, and E. coli). Some of the active bacteria were members of phyla Actinobacteria and Proteobacteria; however, most of the isolates belong to Bacteroidetes, while others could not be assigned to any classification based on $16 \mathrm{~S}$ rRNA analysis [55]. 


\section{Antarctic Bacterial Strains Exhibit Activity against Microorganisms of Industrial Importance}

Unlike the inhibitors produced by mesophylls, the antimicrobials produced in cold environments, such as the Antarctic, need to function at low temperatures for the organisms to gain a competitive advantage during their growth cycle. Such cold-active antimicrobial compounds may be exploitable in industrial applications, including chilled-food preservation [17].

Furthermore, Antarctic bacterial isolates have shown potential against biofilm formation, which is of great importance for different industrial applications. For example, Leyton et al. [70] reported that $26.9 \%$ of Antarctic bacterial isolates (from a total of 67) strongly inhibited the biofilm of Flavobacterium psychrophilum, with the greatest effect shown by proteins $(48-56 \mathrm{kDa})$ secreted by Pseudomonas fragi strains. F. psychrophilum affects farmed salmon and trout worldwide and its ability to form biofilms, in addition to the emergence of resistant strains, have increased the resistance to antibiotics [70].

Additionally, Antarctic antibiotic producers could inhibit other nonhuman pathogens, including plant pathogens. A Bacillus sp. strain, belonging to the phylum Firmicutes, was isolated from Antarctic seawater and showed strong antifungal activity against several plant pathogenic fungi, including Paecilomyces variotii, Colletotrichum gloeosporioides, Fusarium oxysporum, Trichoderma viride, Rhizoctonia solani Kühn, Alternaria longipes, and Sclerotinia sclerotiorum [73]. Some Bacillus species, other than Antarctic and marine, have been reported to produce strong active antimicrobial compounds against phytopathogens [106-108].

Furthermore, Nocardioides sp. belonging to Actinobacteria phylum isolated from Antarctic soil showed extracellular antimicrobial activity against Xanthomonas oryzae, which is one of the most harmful diseases of rice, causing bacterial blight [59]. Similar results against these phytopathogens have been reported for other Actinobacteria isolates (Arthrobacter sp.), Proteobacteria (Pseudomonas sp. and Burkholderia sp.), and Firmicutes strains (Sporosarcina sp.) [56]. Additionally, at least three Halomonas sp. strains isolated from Antarctic sea water showed activity against multiple phytophatogen species, including Xanthomonas axonopodis, Xanthomonas albilineans, Erwinia stewartii, and Erwinia amylovorans [63]. This indicates that Antarctic strains represent a valuable and almost unexplored source of bioactive molecules for biocontrol of plant diseases, representing a worldwide need.

\section{Recent Advances Identified New Antibiotic Molecules from Antarctic Bacteria}

Although just a limited number of antibiotic molecules from Antarctica bacteria have been purified and described, most of them correspond to new molecules (Figure 1). In a study reporting an analysis of a total set of six antimicrobial-producing bacteria by high-resolution mass spectrometry, the resulting exact mass of the purified extracts did not match any known compound in the natural products library for any of the analyzed strains [55]. In addition, some of the purified fractions could not be associated with complete antimicrobial activity, suggesting that one Antarctic isolate may represent a source of numerous new antimicrobial compounds, which might act synergistically against competing microorganisms. Furthermore, the production of methylamine by P. haloplanktis TAC125, with anti-Bcc activity, was demonstrated by VOCs capture, accumulation, and storage, followed by solid-phase microextraction gas chromatography-mass spectrometry (SPME-GC-MS). Despite this finding, methylamine only partially contributes to the effect against Bcc; thus, the efficiency of marine Antarctic bacteria to inhibit almost all Bcc strains may be related to the synergistic effect of multiple unknown VOCs [68].

Additionally, an antibacterial molecule from the Antarctic Cyanobacterium Nostoc sp. was elucidated based on UV, IR, NMR, electron impact mass spectra (EIMS), and electronspray ionization mass spectra (ESIMS) data [72]. The structure of the active principle was proposed as 4-[(5-carboxy2-hydroxy)-benzyl]-1,10-dihydroxy-3,4,7,11,11 pentamethyloctahydrocyclopenta <a $>$ naphthalene, which is an intracellular biomolecule with broad-range antibacterial activity. This molecule is similar to anthraquinone and indane derivatives of a diterpenoid and different from the various biomolecules reported from Nostoc species other than of Antarctic origin. The rate of production of the active principle corresponds to $1.70 \mathrm{mg} \cdot \mathrm{g}^{-1}$ biomass dry weight [72]. 
Another elucidated antibacterial compound structure corresponded to a chromophore 3-amino-1,8-dimethyl-2-phenoxazone-4,5-dicarboxylic acid connected with two cyclopentapeptides, which belongs to actinomycins and was produced by Streptomyces flavovirens, isolated from Antarctic soil samples, with antimicrobial and antitumor activity [23]. Additionally, Asencio et al. [61] reported the presence of violacein in an antibiotic ethanolic extract from Janthinobacterium sp., which also contains other undetermined compounds that can act as synergistic agents with violacein. The pigmented compound of the other Antarctic Janthinobacterium sp. strain was determined by NMR as (3-[5-(3-hydroxyl-1H-indol-3yl)-2-R1-1H-pyrrol-3-ylidene]-2-R1-1H-indol), with some differences for other violacein pigments previously reported [62]. In the same study, the structure of additional pigment obtained from Antarctic Flavobacterium sp. was elucidated and described as the first reported flexirubin with antimicobacterial activity [62].

(a)<smiles>Oc1ccc2[nH]cc(C3=CC(=C4Nc5ccccc54)[Y1]4[nH][nH]c43)c2c1</smiles>

(c)

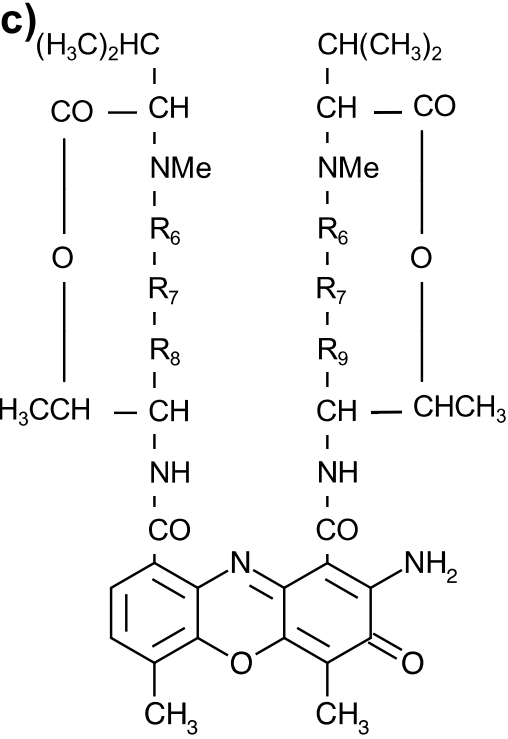

(f)<smiles>CC=CC=[Te]</smiles><smiles>CCC(CC(=O)OC(C)CC(=O)O)OC1OC(C)C(O)C(O)C1O</smiles>

(b)<smiles>[R]c1cc(O)c([R])c(OC(=O)Bc2ccc(O)c([R])c2)c1</smiles>

(d)<smiles>CC1CC(O)C2=C(CCC3(C)C2CC(O)C3(C)C)C1(C)Cc1cc(C(=O)O)ccc1O</smiles>

(e)<smiles>CC(CC(=O)O)OC(=O)C[C@@H](C)OC1OC(C)C(O)C(O)C1O</smiles>

Figure 1. Chemical structures of molecules with antibiotic activity obtained from Antarctic bacteria: (a) Streptomyces flavovirens, (b) and (c) Flavobacterium sp., (d) Nostoc sp., and (e-g) Pseudomonas sp. All molecules, except (e), correspond to novel antimicrobial metabolites. Modified from: [23,54,62,72]. 
Three other soluble compounds from Pseudomonas sp. strain with activity against Bcc strains and S. aureus were elucidated by high-resolution electrospray ionization mass spectrometry (HRESIMS), NMR, and LC-MS as rhamnolipids as follow: (1) $\mathrm{C}_{28} \mathrm{H}_{52} \mathrm{O}_{9}$, containing two fully saturated lipid chains; (2) $\mathrm{C}_{28} \mathrm{H}_{50} \mathrm{O}_{9}$, with one ester, one carboxylic acid group, two olefinic carbons, an anomeric carbon, $\mathrm{A}$ and $\mathrm{B}$ chains having 10 and 12 carbons, respectively, and a single unsaturation at B5; and (3) $\mathrm{C}_{30} \mathrm{H}_{54} \mathrm{O}_{9}$, with $\mathrm{A}$ and $\mathrm{B}$ chains and a single unsaturation at $\mathrm{B} 7$ [54]. The first compound was already known [109], but the last two compounds were reported as new rhamnolipids with antimicrobial activity. Rhamnolipids are well-known secondary metabolites synthesized by members of different Gram negative species. They perform several functions in bacteria, including surface motility and biofilm development [110]. Recently, they have emerged as potential antimicrobials against a broad range of pathogens, due to their intercalation into biological membranes leading to cell death by a permeabilizing effect [111].

\section{Concluding Remarks}

Bacteria isolated from the Antarctic environment are an unexploited source of novel antimicrobial compounds, which may be advantageous in food, therapeutic, health, and industrial applications in the future. Data about Antarctic microorganisms producing antimicrobial compounds are scant in comparison to other extensively studied environments. Although we exposed numerous bacterial strains from different phyla with various antibiotic activity, it is difficult to evaluate and compare the data generated from the different authors, due to the diversity of variable parameters in the screening process, such as isolation circumstances, target selection, and assay conditions. Consequently, it is important to increase investigations focused on antimicrobial activity from Antarctic bacteria in order to amplify comparable available data.

Despite most reports showing Antarctic bacterial strains as a source of antibiotic compounds, the samples/isolates lacked large-scale, high-throughput bioprospection, showing results only for a limited panel of target pathogens and usually just one condition-crude extract. Therefore, developing and applying robust screening models and high-throughput methods is of great importance for studying antimicrobial activity from single or multiple Antarctic bacterial strains in order to increase the chances for the discovery and identification of novel antibiotic molecules. To achieve this goal, it is imperative that the experimental design include all possible variables, such as recovering intra- and extracellular extracts produced in different growth conditions, using possible inducers of antimicrobial activity, and testing against a larger number of targets. To our knowledge, just one study exists where a robust screening model was applied, focusing on the search of antimicrobial activity from Antarctic bacteria, which analyzed 6348 extract samples that were tested in two or three media with different incubation periods and extraction methods [55].

The search for Antarctic microorganisms that could have industrial applications started only in the late 20th century and relatively few scientific groups are pursuing this research. Since then, only a few antibiotic molecules have been purified and elucidated; however, most of them correspond to new molecules. As a result, further work is needed for the identification and purification of new strains, and characterization of the chemical structures of new antibiotics with pharmacological or industrial applications. Geographical locations already covered by research in the Antarctic are limited (Figure 2), particularly on the west side of the continent, and a clear-cut correlation is lacking between the antimicrobial activity pattern and taxonomical distribution of bacterial strains in the Antarctic regions [55]. Thus, there is a great opportunity to continue the discovery and characterization of new antimicrobials from unexplored Antarctic regions. Furthermore, this review does not consider reports of bacteria with antimicrobial activity isolated from Antarctic organisms. Nevertheless, there are reports of their ability to inhibit pathogens, for example for bacteria isolated from sponges [112-114] and macroalgae [115]. Therefore, there could be other unusual habitats within the Antarctic that are suitable for the discovery of antimicrobial compounds. 


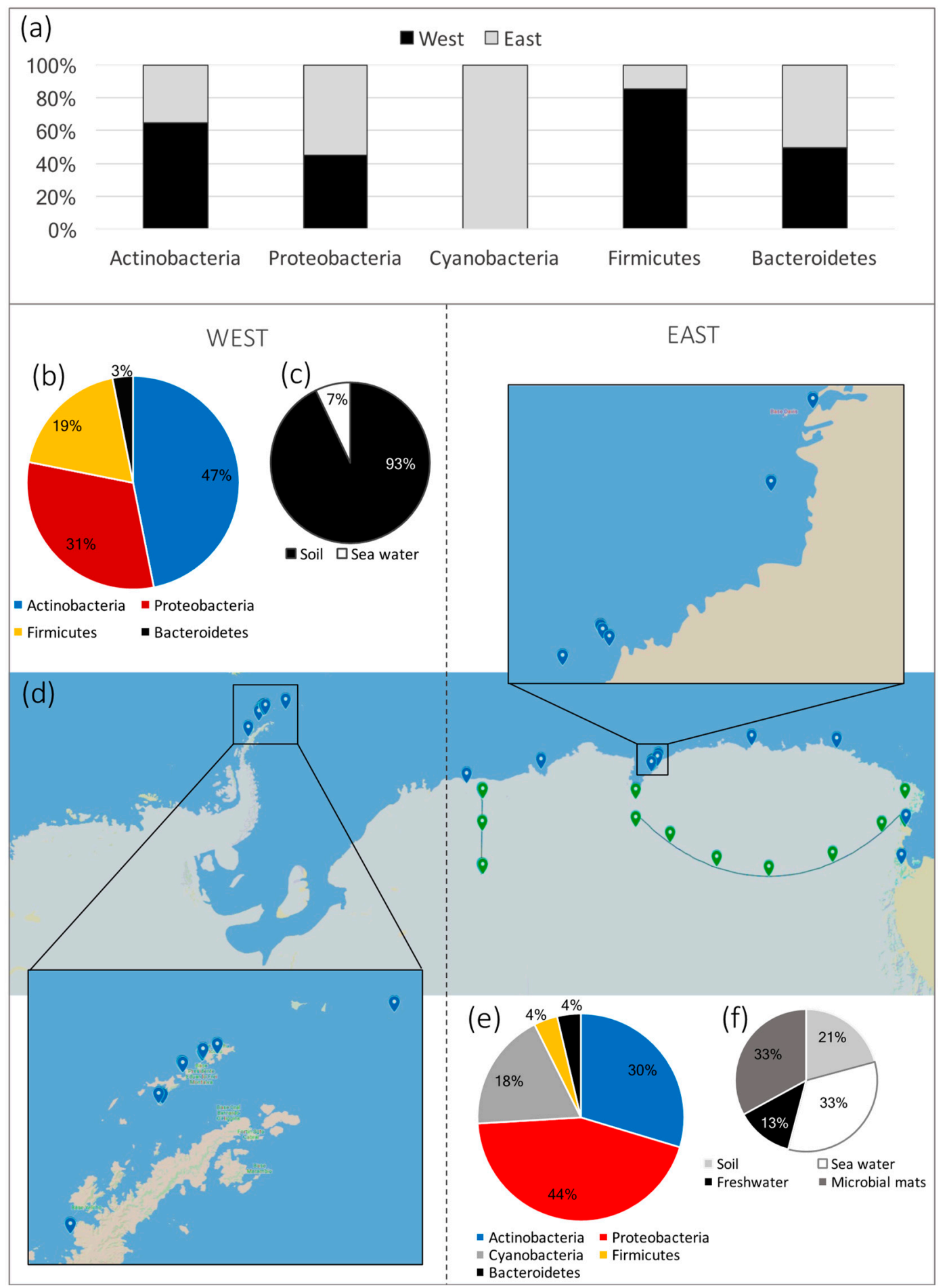

Figure 2. Reported diversity of Antarctic bacteria producing antimicrobial compounds and the geographical locations where diverse sample types were collected for isolation. Different phyla diversity has been reported for west and east Antarctic (a); each phylum has been reported with different frequency for the west side (b) compared to the east counterpart (e). Geographical locations where bacteria with antimicrobial activity have been isolated at each Antarctic side are shown, indicating that the west side has been less explored (d). Blue marks represent regions where punctual sampling was executed; green marks joined by a line illustrate two studies $[14,17]$ where the complete area was sampled; the amplified squares show regions with multiple sampling sites. Active strains have been isolated utilizing different sample sources for west (c) and east (f) continent sides, including soil and seawater for both areas, and freshwater and microbial mats on the west side. 
Finally, we have demonstrated the importance of better research on the inhibitory power of Antarctic strains from a clinical and industrial point of view, but additional Antarctic bioprospecting is necessary in order to understand the role of novel antibacterial molecules in structuring microbial, polar communities from an ecological point of view. There is little information available on the interactions between organisms in polar ecosystems, and very little is known in particular about the microbial diversity and functional capacity of the Antarctic permafrost compared to the Arctic, where the majority of comprehensive metagenomic studies have been done [116]. The application of -omics methods on Antarctic systems will provide an important understanding of microbial diversity, functional capacity of the ecosystem, novel antibiotic molecules, and their role in microbial community modulation. Moreover, as recently highlighted by Mocali et al. [88], multi-omics analyses are needed to generate information that could contribute to filling the gap between genotypic and phenotypic features relating to bacterial cold-adaptation mechanisms. In conclusion, research on Antarctic bacterial strains represents an important potential for biotechnology, while providing a better understanding of polar ecosystems.

Author Contributions: Conceptualization K.N.-M.; Writing-Original Draft Preparation, K.N.-M.; WritingReview\&Editing, K.N.-M. and L.B.; Visualization, K.N.-M.; Supervision, L.B.; Project Administration, L.B.; Funding Acquisition, K.N.-M. and L.B.

Funding: This research was funded by the Chilean National Commission for Scientific and Technological Research [CONICYT-PFCHA/Doctorado Nacional/2017-21170263 to K.N-M], and by Universidad de La Frontera, Grant DI17-0116 and partially funded by Network for Extreme Environments Research (NEXER) Grant.

Acknowledgments: We thank Judith Hoffman from Northern Light Translations for English revision of this review.

Conflicts of Interest: The authors declare no conflict of interest.

\section{References}

1. Livermore, D.M. Has the era of untreatable infections arrived? J. Antimicrob. Chemother. 2009, 64. [CrossRef] [PubMed]

2. Roca, I.; Akova, M.; Baquero, F.; Carlet, J.; Cavaleri, M.; Coenen, S.; Cohen, J.; Findlay, D.; Gyssens, I.; Heure, O.E.; et al. The global threat of antimicrobial resistance: Science for intervention. New Microbes New Infect. 2015, 6, 22-29. [CrossRef] [PubMed]

3. Hughes, D.; Andersson, D.I. Environmental and genetic modulation of the phenotypic expression of antibiotic resistance. FEMS Microbiol. Rev. 2017, 41, 374-391. [CrossRef] [PubMed]

4. Genilloud, O. The re-emerging role of microbial natural products in antibiotic discovery. Antonie van Leeuwenhoek 2014, 106, 173-188. [CrossRef] [PubMed]

5. Bax, R.; Mullan, N.; Verhoef, J. The millennium bugs-The need for and development of new antibacterials. Int. J. Antimicrob. Agents 2000, 16, 51-59. [CrossRef]

6. Axenov-Gribanov, D.V.; Voytsekhovskaya, I.V.; Tokovenko, B.T.; Protasov, E.S.; Gamaiunov, S.V.; Rebets, Y.V.; Luzhetskyy, A.N.; Timofeyev, M.A. Actinobacteria isolated from an underground lake and moonmilk speleothem from the biggest conglomeratic karstic cave in Siberia as sources of novel biologically active compounds. PLoS ONE 2016, 11. [CrossRef] [PubMed]

7. Newman, D.J.; Cragg, G.M.; Snader, K.M. Natural products as sources of new drugs over the period 1981-2002. J. Nat. Prod. 2003, 66, 1022-1037. [CrossRef] [PubMed]

8. Katz, L.; Baltz, R.H. Natural product discovery: Past, present, and future. J. Ind. Microbiol. Biotechnol. 2016, 43, 155-176. [CrossRef] [PubMed]

9. Onaka, H. Novel antibiotic screening methods to awaken silent or cryptic secondary metabolic pathways in actinomycetes. J. Antibiot. 2017, 70, 865-870. [CrossRef] [PubMed]

10. Bredholt, H.; Fjærvik, E.; Johnsen, G.; Zotchev, S.B. Actinomycetes from sediments in the Trondheim fjord, Norway: Diversity and biological activity. Mar. Drugs 2008, 6, 12-24. [CrossRef] [PubMed]

11. Lee, L.H.; Cheah, Y.K.; Nurul Syakima, A.M.; Shiran, M.S.; Tang, Y.L.; Lin, H.P.; Hong, K. Analysis of Antarctic protobacteria by PCR fingerprinting and screening for antimicrobial secondary metabolites. Genet. Mol. Res. 2012, 11, 1627-1641. [CrossRef] [PubMed] 
12. Lam, K.S. New aspects of natural products in drug discovery. Trends Microbiol. 2007, 15, 279-289. [CrossRef] [PubMed]

13. Donadio, S.; Maffioli, S.; Monciardini, P.; Sosio, M.; Jabes, D. Antibiotic discovery in the twenty-first century: Current trends and future perspectives. J. Antibiot. 2010, 63, 423-430. [CrossRef] [PubMed]

14. Lo Giudice, A.; Bruni, V.; Michaud, L. Characterization of Antarctic psychrotrophic bacteria with antibacterial activities against terrestrial microorganisms. J. Basic Microbiol. 2007, 47, 496-505. [CrossRef] [PubMed]

15. Gulder, T.A.; Moore, B.S. Chasing the treasures of the sea-Bacterial marine natural products. Curr. Opin. Microbiol. 2009, 12, 252-260. [CrossRef] [PubMed]

16. Land, M.; Hauser, L.; Jun, S.-R.; Nookaew, I.; Leuze, M.R.; Ahn, T.-H.; Karpinets, T.; Lund, O.; Kora, G.; Wassenaar, T.; et al. Insights from 20 years of bacterial genome sequencing. Funct. Integr. Genom. 2015, 15, 141-161. [CrossRef] [PubMed]

17. O'Brien, A.; Sharp, R.; Russell, N.J.; Roller, S. Antarctic bacteria inhibit growth of food-borne microorganisms at low temperatures. FEMS Microbiol. Ecol. 2004, 48, 157-167. [CrossRef] [PubMed]

18. Undabarrena, A.; Beltrametti, F.; Claverías, F.P.; González, M.; Moore, E.R.B.; Seeger, M.; Cámara, B. Exploring the diversity and antimicrobial potential of marine actinobacteria from the comau fjord in Northern Patagonia, Chile. Front. Microbiol. 2016, 7. [CrossRef] [PubMed]

19. Mehetre, G.; Shah, M.; Dastager, S.G.; Dharne, M.S. Untapped bacterial diversity and metabolic potential within Unkeshwar hot springs, India. Arch. Microbiol. 2018, 200, 753-770. [CrossRef] [PubMed]

20. Jiang, W.; Zhong, Y.; Shen, L.; Wu, X.; Ye, Y.; Chen, C.-T.; Wu, B. Stress-driven discovery of natural products from extreme marine environment- kueishantao hydrothermal vent, a case study of metal switch valve. Curr. Org. Chem. 2014, 18. [CrossRef]

21. Yogabaanu, U.; Weber, J.F.F.; Convey, P.; Rizman-Idid, M.; Alias, S.A. Antimicrobial properties and the influence of temperature on secondary metabolite production in cold environment soil fungi. Polar Sci. 2017, 14, 60-67. [CrossRef]

22. Lewis Smith, R.I. Exotic sporomorpha as indicators of potential immigrant colonists in antarctica. Grana 1991, 30, 313-324. [CrossRef]

23. Bratchkova, A.; Ivanova, V. Bioactive metabolites produced by microorganisms collected in Antarctica and the Arctic. Biotechnol. Biotechnol. Equip. 2011, 25, 1-7. [CrossRef]

24. Wynn-Williams, D.D. Ecological aspects of Antarctic microbiology. Adv. Microb. Ecol. 1990, 11, 71-146.

25. Convey, P.; Gibson, J.A.E.; Hillenbrand, C.D.; Hodgson, D.A.; Pugh, P.J.A.; Smellie, J.L.; Stevens, M.I. Antarctic terrestrial life-Challenging the history of the frozen continent? Biol. Rev. 2008, 83, 103-117. [CrossRef] [PubMed]

26. Corsolini, S. Industrial contaminants in Antarctic biota. J. Chromatogr. A 2009, 1216, 598-612. [CrossRef] [PubMed]

27. Lo Giudice, A.; Casella, P.; Bruni, V.; Michaud, L. Response of bacterial isolates from Antarctic shallow sediments towards heavy metals, antibiotics and polychlorinated biphenyls. Ecotoxicology 2013, 22, 240-250. [CrossRef] [PubMed]

28. Cowan, D.A.; Chown, S.L.; Convey, P.; Tuffin, M.; Hughes, K.; Pointing, S.; Vincent, W.F. Non-indigenous microorganisms in the Antarctic: Assessing the risks. Trends Microbiol. 2011, 19, 540-548. [CrossRef] [PubMed]

29. Martins, C.C.; Aguiar, S.N.; Wisnieski, E.; Ceschim, L.M.M.; Figueira, R.C.L.; Montone, R.C. Baseline concentrations of faecal sterols and assessment of sewage input into different inlets of Admiralty Bay, King George Island, Antarctica. Mar. Pollut. Bull. 2014, 78, 218-223. [CrossRef] [PubMed]

30. Zreda-Gostynska, G.; Kyle, P.R.; Finnegan, D.; Prestbo, K.M. Volcanic gas emissions from Mount Erebus and their impact on the Antarctic environment. J. Geophys. Res. Solid Earth 1997, 102, 15039-15055. [CrossRef]

31. Bargagli, R. Environmental contamination in Antarctic ecosystems. Sci. Total Environ. 2008, 400, $212-226$. [CrossRef] [PubMed]

32. Cipro, C.V.Z.Z.; Yogui, G.T.; Bustamante, P.; Taniguchi, S.; Sericano, J.L.; Montone, R.C. Organic pollutants and their correlation with stable isotopes in vegetation from King George Island, Antarctica. Chemosphere 2011, 85, 393-398. [CrossRef] [PubMed]

33. Nichols, D.; Bowman, J.; Sanderson, K.; Nichols, C.M.; Lewis, T.; McMeekin, T.; Nichols, P.D. Developments with Antarctic microorganisms: Culture collections, bioactivity screening, taxonomy, PUFA production and cold-adapted enzymes. Curr. Opin. Biotechnol. 1999, 10, 240-246. [CrossRef] 
34. Aislabie, J.M.; Jordan, S.; Barker, G.M. Relation between soil classification and bacterial diversity in soils of the Ross Sea region, Antarctica. Geoderma 2008, 144, 9-20. [CrossRef]

35. Cary, S.C.; McDonald, I.R.; Barrett, J.E.; Cowan, D.A. On the rocks: The microbiology of Antarctic Dry Valley soils. Nat. Rev. Microbiol. 2010, 8, 129-138. [CrossRef] [PubMed]

36. Loperena, L.; Soria, V.; Varela, H.; Lupo, S.; Bergalli, A.; Guigou, M.; Pellegrino, A.; Bernardo, A.; Calviño, A.; Rivas, F.; et al. Extracellular enzymes produced by microorganisms isolated from maritime Antarctica. World J. Microbiol. Biotechnol. 2012, 28, 2249-2256. [CrossRef] [PubMed]

37. Pearce, D.A.; Newsham, K.K.; Thorne, M.A.S.; Calvo-Bado, L.; Krsek, M.; Laskaris, P.; Hodson, A.; Wellington, E.M. Metagenomic Analysis of a Southern Maritime Antarctic Soil. Front. Microbiol. 2012, 3. [CrossRef] [PubMed]

38. Silva, T.R.; Duarte, A.W.F.; Passarini, M.R.Z.; Ruiz, A.L.T.G.; Franco, C.H.; Moraes, C.B.; de Melo, I.S.; Rodrigues, R.A.; Fantinatti-Garboggini, F.; Oliveira, V.M. Bacteria from Antarctic environments: Diversity and detection of antimicrobial, antiproliferative, and antiparasitic activities. Polar Biol. 2018, 41, 1505-1519. [CrossRef]

39. Singh, B.K.; Munro, S.; Potts, J.M.; Millard, P. Influence of grass species and soil type on rhizosphere microbial community structure in grassland soils. Appl. Soil Ecol. 2007, 36, 147-155. [CrossRef]

40. Papale, M.; Conte, A.; Mikkonen, A.; Michaud, L.; La Ferla, R.; Azzaro, M.; Caruso, G.; Paranhos, R.; Cabral Anderson, S.; Maimone, G.; et al. Prokaryotic assemblages within permafrost active layer at Edmonson Point (Northern Victoria Land, Antarctica). Soil Biol. Biochem. 2018, 123, 165-179. [CrossRef]

41. Shivaji, S.; Reddy, G.S.; Aduri, R.P.; Kutty, R.; Ravenschlag, K. Bacterial diversity of a soil sample from Schirmacher Oasis, Antarctica. Cell. Mol. Biol. 2004, 50, 525-536. [PubMed]

42. Teixeira, L.C.R.S.; Peixoto, R.S.; Cury, J.C.; Sul, W.J.; Pellizari, V.H.; Tiedje, J.; Rosado, A.S. Bacterial diversity in rhizosphere soil from Antarctic vascular plants of Admiralty Bay, maritime Antarctica. Isme J. 2010, 4, 989. [CrossRef] [PubMed]

43. Marizcurrena, J.J.; Morel, M.A.; Braña, V.; Morales, D.; Martinez-López, W.; Castro-Sowinski, S. Searching for novel photolyases in UVC-resistant Antarctic bacteria. Extremophiles 2017, 21, 409-418. [CrossRef] [PubMed]

44. Lamilla, C.; Pavez, M.; Santos, A.; Hermosilla, A.; Llanquinao, V.; Barrientos, L. Bioprospecting for extracellular enzymes from culturable Actinobacteria from the South Shetland Islands, Antarctica. Polar Biol. 2017, 40, 719-726. [CrossRef]

45. Subramani, R.; Aalbersberg, W. Culturable rare Actinomycetes: Diversity, isolation and marine natural product discovery. Appl. Microbiol. Biotechnol. 2013, 97, 9291-9321. [CrossRef] [PubMed]

46. Tribelli, P.M.; Rossi, L.; Ricardi, M.M.; Gomez-Lozano, M.; Molin, S.; Raiger Iustman, L.J.; Lopez, N.I. Microaerophilic alkane degradation in Pseudomonas extremaustralis: A transcriptomic and physiological approach. J. Ind. Microbiol. Biotechnol. 2018, 45, 15-23. [CrossRef] [PubMed]

47. Dube, S.; Singh, L.; Alam, S.I. Proteolytic anaerobic bacteria from lake sediments of Antarctica. Enzyme Microb. Technol. 2001, 28, 114-121. [CrossRef]

48. Mountfort, D.O.; Rainey, F.A.; Burghardt, J.; Kaspar, H.F.; Stackebrandt, E. Clostridium vincentii sp. nov., a new obligately anaerobic, saccharolytic, psychrophilic bacterium isolated from low-salinity pond sediment of the McMurdo Ice Shelf, Antarctica. Arch. Microbiol. 1997, 167, 54-60. [CrossRef] [PubMed]

49. Bowman, J.P.; Cavanagh, J.; Austin, J.J.; Sanderson, K. Novel Psychrobacter species from Antarctic ornithogenic soils. Int. J. Syst. Bacteriol. 1996, 46, 841-848. [CrossRef] [PubMed]

50. Vishniac, H. Soil microgeology. In Antarctic Microbiology; Friedmann, Ed.; Wiley-Liss: New York, NY, USA, 1993; pp. 297-341.

51. Long, R.A.; Azam, F. Antagonistic Interactions among Marine Pelagic Bacteria. Appl. Environ. Microbiol. 2001, 67, 4975-4983. [CrossRef] [PubMed]

52. Nair, S.; Simidu, U. Distribution and significance of heterotrophic marine bacteria with antibacterial activity. Appl. Environ. Microbiol. 1987, 53, 2957-2962. [PubMed]

53. Gram, L.; Grossart, H.P.; Schlingloff, A.; Kiørboe, T. Possible quorum sensing in marine snow bacteria: Production of acylated homoserine lactones by Roseobacter strains isolated from marine snow. Appl. Environ. Microbiol. 2002, 68, 4111-4116. [CrossRef] [PubMed]

54. Tedesco, P.; Maida, I.; Esposito, F.P.; Tortorella, E.; Subko, K.; Ezeofor, C.C.; Zhang, Y.; Tabudravu, J.; Jaspars, M.; Fani, R.; et al. Antimicrobial activity of monoramnholipids produced by bacterial strains isolated from the Ross Sea (Antarctica). Mar. Drugs 2016, 14, 83. [CrossRef] [PubMed] 
55. Rojas, J.L.; Martín, J.; Tormo, J.R.; Vicente, F.; Brunati, M.; Ciciliato, I.; Losi, D.; Van Trappen, S.; Mergaert, J.; Swings, J.; et al. Bacterial diversity from benthic mats of Antarctic lakes as a source of new bioactive metabolites. Mar. Genom. 2009, 2, 33-41. [CrossRef] [PubMed]

56. Tomova, I.; Stoilova-Disheva, M.; Lazarkevich, I.; Vasileva-Tonkova, E. Antimicrobial activity and resistance to heavy metals and antibiotics of heterotrophic bacteria isolated from sediment and soil samples collected from two Antarctic islands. Front. Life Sci. 2015, 8, 348-357. [CrossRef]

57. Lee, L.H.; Cheah, Y.K.; Sidik, S.M.; Mutalib, N.S.A.; Tang, Y.L.; Lin, H.P.; Hong, K. Molecular characterization of Antarctic actinobacteria and screening for antimicrobial metabolite production. World J. Microbiol. Biotechnol. 2012, 28, 2125-2137. [CrossRef] [PubMed]

58. Cheah, Y.K.; Lee, L.H.; Chieng, C.Y.C.; Wong, V.L.C.M. Isolation, identification and screening of actinobacteria in volcanic soil of deception island (the Antarctic) for antimicrobial metabolites. Polish Polar Res. 2015, 36, 67-78. [CrossRef]

59. Gesheva, V.; Vasileva-Tonkova, E. Production of enzymes and antimicrobial compounds by halophilic Antarctic Nocardioides sp. grown on different carbon sources. World J. Microbiol. Biotechnol. 2012, 28, 2069-2076. [CrossRef] [PubMed]

60. Lavin, P.L.; Yong, S.T.; Wong, C.M.V.L.; De Stefano, M. Isolation and characterization of Antarctic psychrotroph Streptomyces sp. strain INACH3013. Antarct. Sci. 2016, 28, 433-442. [CrossRef]

61. Asencio, G.; Lavin, P.; Alegría, K.; Domínguez, M.; Bello, H.; González-Rocha, G.; González-Aravena, M. Antibacterial activity of the Antarctic bacterium Janthinobacterium sp. SMN 33.6 against multi-resistant Gram-negative bacteria. Electron. J. Biotechnol. 2014, 17, 1-5. [CrossRef]

62. Mojib, N.; Philpott, R.; Huang, J.P.; Niederweis, M.; Bej, A.K. Antimycobacterial activity in vitro of pigments isolated from Antarctic bacteria. Antonie van Leeuwenhoek 2010, 98, 531-540. [CrossRef] [PubMed]

63. Danilovich, M.E.; Sánchez, L.A.; Acosta, F.; Delgado, O.D. Antarctic bioprospecting: In pursuit of microorganisms producing new antimicrobials and enzymes. Polar Biol. 2018, 47, 1417-1433. [CrossRef]

64. Fukuda, W.; Kimura, T.; Araki, S.; Miyoshi, Y.; Atomi, H.; Imanaka, T. Lysobacter oligotrophicus sp. nov., isolated from an Antarctic freshwater lake in Antarctica. Int. J. Syst. Evol. Microbiol. 2013, 63, 3313-3318. [CrossRef] [PubMed]

65. Maida, I.; Bosi, E.; Fondi, M.; Perrin, E.; Orlandini, V.; Papaleo, M.C.; Mengoni, A.; de Pascale, D.; Tutino, M.L.; Michaud, L.; et al. Antimicrobial activity of Pseudoalteromonas strains isolated from the Ross Sea (Antarctica) versus Cystic Fibrosis opportunistic pathogens. Hydrobiologia 2015, 761, 443-457. [CrossRef]

66. Papa, R.; Parrilli, E.; Sannino, F.; Barbato, G.; Tutino, M.L.; Artini, M.; Selan, L. Anti-biofilm activity of the Antarctic marine bacterium Pseudoalteromonas haloplanktis TAC125. Res. Microbiol. 2013, 164, 450-456. [CrossRef] [PubMed]

67. Médigue, C.; Krin, E.; Pascal, G.; Barbe, V.; Bernsel, A.; Bertin, P.N.; Cheung, F.; Cruveiller, S.; D'Amico, S.; Duilio, A.; et al. Coping with cold: The genome of the versatile marine Antarctica bacterium Pseudoalteromonas haloplanktis TAC125. Genome Res. 2005, 15, 1325-1335. [CrossRef] [PubMed]

68. Sannino, F.; Parrilli, E.; Apuzzo, G.A.; de Pascale, D.; Tedesco, P.; Maida, I.; Perrin, E.; Fondi, M.; Fani, R.; Marino, G.; et al. Pseudoalteromonas haloplanktis produces methylamine, a volatile compound active against Burkholderia cepacia complex strains. New Biotechnol. 2017, 35, 13-18. [CrossRef] [PubMed]

69. Wong, C.M.V.L.; Tam, H.K.; Alias, S.A.; González, M.; González-Rocha, G.; Domínguez-Yévenes, M. Pseudomonas and pedobacter isolates from King George Island inhibited the growth of foodborne pathogens. Polish Polar Res. 2011, 32, 3-14. [CrossRef]

70. Leyton, A.; Urrutia, H.; Vidal, J.M.; de la Fuente, M.; Alarcón, M.; Aroca, G.; González-Rocha, G.; Sossa, K. Actividad inhibitoria del sobrenadante de la bacteria Antártica Pseudomonas sp. M19B en la formación de biopelículas de Flavobacterium psychrophilum 19749. Rev. Biol. Mar. Oceanogr. 2015, 50, 375-381. [CrossRef]

71. Taton, A.; Grubisic, S.; Ertz, D.; Hodgson, D.A.; Piccardi, R.; Biondi, N.; Tredici, M.R.; Mainini, M.; Losi, D.; Marinelli, F.; et al. Polyphasic study of antarctic cyanobacterial strains. J. Phycol. 2006, 42, 1257-1270. [CrossRef]

72. Asthana, R.K.; Deepali; Tripathi, M.K.; Srivastava, A.; Singh, A.P.; Singh, S.P.; Nath, G.; Srivastava, R.; Srivastava, B.S. Isolation and identification of a new antibacterial entity from the Antarctic cyanobacterium Nostoc CCC 537. J. Appl. Phycol. 2009, 21, 81-88. [CrossRef]

73. Guo, W.; Cui, P.; Chen, X. Complete genome of Bacillus sp. Pc3 isolated from the Antarctic seawater with antimicrobial activity. Mar. Genom. 2015, 20, 1-2. [CrossRef] [PubMed] 
74. Vollú, R.E.; Jurelevicius, D.; Ramos, L.R.; Peixoto, R.S.; Rosado, A.S.; Seldin, L. Aerobic endospore-forming bacteria isolated from Antarctic soils as producers of bioactive compounds of industrial interest. Polar Biol. 2014, 37, 1121-1131. [CrossRef]

75. Shekh, R.M.; Singh, P.; Singh, S.M.; Roy, U. Antifungal activity of Arctic and Antarctic bacteria isolates. Polar Biol. 2011, 34, 139-143. [CrossRef]

76. Van den Burg, B. Extremophiles as a source for novel enzymes. Curr. Opin. Microbiol. 2003, 6, $213-218$. [CrossRef]

77. Casanueva, A.; Tuffin, M.; Cary, C.; Cowan, D.A. Molecular adaptations to psychrophily: The impact of "omic" technologies. Trends Microbiol. 2010, 18, 374-381. [CrossRef] [PubMed]

78. D'Amico, S.; Collins, T.; Marx, J.C.; Feller, G.; Gerday, C. Psychrophilic microorganisms: Challenges for life. EMBO Rep. 2006, 7, 385-389. [CrossRef] [PubMed]

79. Lo Giudice, A.; Brilli, M.; Bruni, V.; De Domenico, M.; Fani, R.; Michaud, L. Bacterium-bacterium inhibitory interactions among psychrotrophic bacteria isolated from Antarctic seawater (Terra Nova Bay, Ross Sea). FEMS Microbiol. Ecol. 2007, 60, 383-396. [CrossRef] [PubMed]

80. Leiva, S.; Alvarado, P.; Huang, Y.; Wang, J.; Garrido, I. Diversity of pigmented Gram-positive bacteria associated with marine macroalgae from Antarctica. FEMS Microbiol. Lett. 2015, 362. [CrossRef] [PubMed]

81. Cristóbal, H.A.; López, M.A.; Kothe, E.; Abate, C.M. Diversity of protease-producing marine bacteria from sub-antarctic environments. J. Basic Microbiol. 2011, 51, 590-600. [CrossRef] [PubMed]

82. Kim, E.H.; Cho, K.H.; Lee, Y.M.; Yim, J.H.; Lee, H.K.; Cho, J.C.; Hong, S.G. Diversity of cold-active protease-producing bacteria from arctic terrestrial and marine environments revealed by enrichment culture. J. Microbiol. 2010, 48, 426-432. [CrossRef] [PubMed]

83. Lo Giudice, A.; Caruso, C.; Mangano, S.; Bruni, V.; De Domenico, M.; Michaud, L. Marine Bacterioplankton Diversity and Community Composition in an Antarctic Coastal Environment. Microb. Ecol. 2012, 63, $210-223$. [CrossRef] [PubMed]

84. Bosi, E.; Fondi, M.; Orlandini, V.; Perrin, E.; Maida, I.; de Pascale, D.; Tutino, M.L.; Parrilli, E.; Lo Giudice, A.; Filloux, A.; et al. The pangenome of (Antarctic) Pseudoalteromonas bacteria: Evolutionary and functional insights. BMC Genom. 2017, 18. [CrossRef] [PubMed]

85. Weintraub, S.J.; Manson, S.R. Asparagine deamidation: A regulatory hourglass. Mech. Ageing Dev. 2004, 125, 255-257. [CrossRef] [PubMed]

86. Parrilli, E.; Giuliani, M.; Giordano, D.; Russo, R.; Marino, G.; Verde, C.; Tutino, M.L. The role of a 2-on-2 haemoglobin in oxidative and nitrosative stress resistance of Antarctic Pseudoalteromonas haloplanktis TAC125. Biochimie 2010, 92, 1003-1009. [CrossRef] [PubMed]

87. Wilmes, B.; Hartung, A.; Lalk, M.; Liebeke, M.; Schweder, T.; Neubauer, P. Fed-batch process for the psychrotolerant marine bacterium Pseudoalteromonas haloplanktis. Microb. Cell Fact. 2010, 9. [CrossRef] [PubMed]

88. Mocali, S.; Chiellini, C.; Fabiani, A.; Decuzzi, S.; Pascale, D.; Parrilli, E.; Tutino, M.L.; Perrin, E.; Bosi, E.; Fondi, M.; et al. Ecology of cold environments: New insights of bacterial metabolic adaptation through an integrated genomic-phenomic approach. Sci. Rep. 2017, 7. [CrossRef] [PubMed]

89. Dieser, M.; Greenwood, M.; Foreman, C.M. Carotenoid Pigmentation in Antarctic Heterotrophic Bacteria as a Strategy to Withstand Environmental Stresses. Arctic Antarct. Alp. Res. 2010, 42, 396-405. [CrossRef]

90. Lo Giudice, A.; Fani, R. Antimicrobial Potential of Cold-Adapted Bacteria and Fungi from Polar Regions. In Biotechnology of Extremophiles; Springer International Publishing: New York, NY, USA, 2016; pp. 83-115. ISBN 978-3-319-13520-5.

91. Fondi, M.; Bosi, E.; Lo Giudice, A.; Fani, R. A Systems Biology View on Bacterial Response to Temperature Shift. In Biotechnology of Extremophiles; Springer International Publishing: New York, NY, USA, 2016; pp. 597-618. ISBN 978-3-319-13520-5.

92. Holmström, C.; Kjelleberg, S. Marine Pseudoalteromonas species are associated with higher organisms and produce biologically active extracellular agents. FEMS Microbiol. Ecol. 1999, 30, 285-293. [CrossRef]

93. Isnansetyo, A.; Kamei, Y. MC21-A, a bactericidal antibiotic produced by a new marine bacterium, Pseudoalteromonas phenolica sp. nov. O-BC30T, against methicillin-resistant Staphylococcus aureus. Antimicrob. Agents Chemother. 2003, 47, 480-488. [CrossRef] [PubMed] 
94. Xie, B.; Qin, Y.L.S.; Rong, J.C.; Zhang, X.Y.; Chen, X.L.; Zhou, B.C.; Zhang, Y.Z. Genome Sequence of the Cycloprodigiosin-Producing Bacterial Strain Pseudoalteromonas rubra ATCC 29570 T. J. Bacteriol. 2012, 194, 1637-1638. [CrossRef] [PubMed]

95. Holmström, C.; Egan, S.; Franks, A.; McCloy, S.; Kjelleberg, S. Antifouling activities expressed by marine surface associated Pseudoalteromonas species. FEMS Microbiol. Ecol. 2002, 41, 47-58. [CrossRef]

96. Goodfellow, M.; Fiedler, H.P. A guide to successful bioprospecting: Informed by actinobacterial systematics. Antonie van Leeuwenhoek 2010, 98, 119-142. [CrossRef] [PubMed]

97. Sen, A.; Daubin, V.; Abrouk, D.; Gifford, I.; Berry, A.M.; Normand, P.; Philippe Normand, C. Phylogeny of the class Actinobacteria revisited in the light of complete genomes. Int. J. Syst. Evol. Microbiol. 2014, 64, 3821-3832. [CrossRef] [PubMed]

98. Doroghazi, J.R.; Metcalf, W.W. Comparative genomics of actinomycetes with a focus on natural product biosynthetic genes. BMC Genom. 2013, 14. [CrossRef] [PubMed]

99. Okoro, C.K.; Brown, R.; Jones, A.L.; Andrews, B.A.; Asenjo, J.A.; Goodfellow, M.; Bull, A.T. Diversity of culturable actinomycetes in hyper-arid soils of the Atacama Desert, Chile. Antonie van Leeuwenhoek 2009, 95, 121-133. [CrossRef] [PubMed]

100. Thakur, D.; Yadav, A.; Gogoi, B.K.; Bora, T.C. Isolation and screening of Streptomyces in soil of protected forest areas from the states of Assam and Tripura, India, for antimicribial metabolites. J. Mycol. Med. 2007, 17, 242-249. [CrossRef]

101. Fischbach, M.A.; Walsh, C.T. Assembly-line enzymology for polyketide and nonribosomal peptide antibiotics: Logic machinery, and mechanisms. Chem. Rev. 2006, 106, 3468-3496. [CrossRef] [PubMed]

102. Maida, I.; Fondi, M.; Papaleo, M.C.; Perrin, E.; Orlandini, V.; Emiliani, G.; de Pascale, D.; Parrilli, E.; Tutino, M.L.; Michaud, L.; et al. Phenotypic and genomic characterization of the Antarctic bacterium Gillisia sp. CAL575, a producer of antimicrobial compounds. Extremophiles 2014, 18, 35-49. [CrossRef] [PubMed]

103. Vincent, W.F. Cyanobacterial Dominance in the Polar Regions. In The Ecology of Cyanobacteria; Springer: Dordrecht, The Netherlands, 2000; pp. 321-340. ISBN 978-0-7923-4735-4.

104. Zakhia, F.; Jungblut, A.D.; Taton, A.; Vincent, W.F.; Wilmotte, A. Cyanobacteria in cold ecosystems. In Psychrophiles: From Biodiversity to Biotechnology; Springer: Berlin/Heidelberg, Germany, 2008; pp. 121-135. ISBN 9783540743347.

105. Biondi, N.; Tredici, M.R.; Taton, A.; Wilmotte, A.; Hodgson, D.A.; Losi, D.; Marinelli, F. Cyanobacteria from benthic mats of Antarctic lakes as a source of new bioactivities. J. Appl. Microbiol. 2008, 105, 105-115. [CrossRef] [PubMed]

106. Kumar, S.N.; Nambisan, B.; Sundaresan, A.; Mohandas, C.; Anto, R.J. Isolation and identification of antimicrobial secondary metabolites from Bacillus cereus associated with a rhabditid entomopathogenic nematode. Ann. Microbiol. 2014, 64, 209-218. [CrossRef]

107. Li, B.; Li, Q.; Xu, Z.; Zhang, N.; Shen, Q.; Zhang, R. Responses of beneficial Bacillus amyloliquefaciens SQR9 to different soilborne fungal pathogens through the alteration of antifungal compounds production. Front. Microbiol. 2014, 5. [CrossRef] [PubMed]

108. Zhao, Z.; Wang, Q.; Wang, K.; Brian, K.; Liu, C.; Gu, Y. Study of the antifungal activity of Bacillus vallismortis ZZ185 in vitro and identification of its antifungal components. Bioresour. Technol. 2010, 101, $292-297$. [CrossRef] [PubMed]

109. Sharma, A.; Jansen, R.; Nimtz, M.; Johri, B.N.; Wray, V. Rhamnolipids from the rhizosphere bacterium Pseudomonas sp. GRP3that reduces damping-off disease in chilli and tomato nurseries. J. Nat. Prod. 2007, 70, 941-947. [CrossRef] [PubMed]

110. Abdel-Mawgoud, A.M.; Lépine, F.; Déziel, E. Rhamnolipids: Diversity of structures, microbial origins and roles. Appl. Microbiol. Biotechnol. 2010, 86, 1323-1336. [CrossRef] [PubMed]

111. Sotirova, A.V.; Spasova, D.I.; Galabova, D.N.; Karpenko, E.; Shulga, A. Rhamnolipid-biosurfactant permeabilizing effects on gram-positive and gram-negative bacterial strains. Curr. Microbiol. 2008, 56, 639-644. [CrossRef] [PubMed]

112. Papaleo, M.C.; Fondi, M.; Maida, I.; Perrin, E.; Lo Giudice, A.; Michaud, L.; Mangano, S.; Bartolucci, G.; Romoli, R.; Fani, R. Sponge-associated microbial Antarctic communities exhibiting antimicrobial activity against Burkholderia cepacia complex bacteria. Biotechnol. Adv. 2012, 30, 272-293. [CrossRef] [PubMed] 
113. Papaleo, M.C.; Romoli, R.; Bartolucci, G.; Maida, I.; Perrin, E.; Fondi, M.; Orlandini, V.; Mengoni, A.; Emiliani, G.; Tutino, M.L.; et al. Bioactive volatile organic compounds from Antarctic (sponges) bacteria. New Biotechnol. 2013, 30, 824-838. [CrossRef] [PubMed]

114. Thomas, T.R.A.; Kavlekar, D.P.; LokaBharathi, P.A. Marine drugs from sponge-microbe association-A review. Mar. Drugs 2010, 8, 1417-1468. [CrossRef] [PubMed]

115. Alvarado, P.; Huang, Y.; Wang, J.; Garrido, I.; Leiva, S. Phylogeny and bioactivity of epiphytic Gram-positive bacteria isolated from three co-occurring antarctic macroalgae. Antonie Van Leeuwenhoek 2018, 1-13. [CrossRef] [PubMed]

116. Makhalanyane, T.P.; Van Goethem, M.W.; Cowan, D.A. Microbial diversity and functional capacity in polar soils. Curr. Opin. Biotechnol. 2016, 38, 159-166. [CrossRef] [PubMed]

2018 by the authors. Licensee MDPI, Basel, Switzerland. This article is an open access article distributed under the terms and conditions of the Creative Commons Attribution (CC BY) license (http:/ / creativecommons.org/licenses/by/4.0/). 